\title{
Gate- and Light-Tunable Negative Differential Resistance with High Peak Current Density in $1 \mathrm{~T}-\mathrm{TaS}_{2} / 2 \mathrm{H}-\mathrm{MoS}_{2} \mathrm{~T}$-Junction
}

\author{
Mehak Mahajan and Kausik Majumdar* \\ Department of Electrical Communication Engineering, Indian Institute of Science, \\ Bangalore 560012, India \\ E-mail: kausikm@iisc.ac.in
}

\begin{abstract}
Metal-based electronics is attractive for fast and radiation-hard electronic circuits and remains one of the longstanding goals for researchers. The emergence of $1 \mathrm{~T}-\mathrm{TaS}_{2}$, a layered material exhibiting strong charge density wave (CDW) driven resistivity switching that can be controlled by an external stimulus such as electric field and optical pulses, has triggered a renewed interest in metal-electronics. Here we demonstrate a negative differential resistor (NDR) using electrically driven CDW phase transition in an asymmetrically designed T-junction made up of $1 \mathrm{~T}-\mathrm{TaS}_{2} / 2 \mathrm{H}-\mathrm{MoS}_{2}$ van der Waals heterostructure. The principle of operation of the proposed device is governed by majority carrier transport and is distinct from usual NDR devices employing tunneling of carriers, thus avoids the bottleneck of weak tunneling efficiency in van der Waals heterojunctions. Consequently, we achieve a peak current density in excess of $10^{5} \mathrm{nA} \mu \mathrm{m}^{-2}$, which is
\end{abstract}


about two orders of magnitude higher than that obtained in typical layered material based NDR implementations. The peak current density can be effectively tuned by an external gate voltage as well as photo-gating. The device is robust against ambiance-induced degradation and the characteristics repeat in multiple measurements over a period of more than a month. The findings are attractive for the implementation of active metal-based functional circuits.

Keywords: $1 \mathrm{~T}-\mathrm{TaS}_{2}, \mathrm{MoS}_{2}$, charge density wave, van der Waals heterojunction, negative differential resistance, peak-to-valley current ratio, photo-gating 
Metal-based electronics remains one of the longstanding goals of researchers to achieve ultra-fast and radiation-hard electronic circuits. ${ }^{115}$ In modern electronics, metals are primarily used as passive conductors and usually do not play any active role. Nanoscale materials with their distinctive size dependent properties provide opportunities to achieve unexplored device functionalities. Ta-based di-chalcogenides, which form layered structures and exhibit charge density wave (CDW),$\underline{617}$ are particularly promising in the context. For example, it has been recently shown that $2 \mathrm{H}-\mathrm{TaSe}_{2}$, while showing excellent conductivity properties, also exhibits strong photoluminescence and long photo-carrier lifetime, allowing active metal-based device applications. ${ }^{8}$ CDW is a macroscopic state which is exhibited by materials with reduced dimension, for example, one-dimensional and layered two-dimensional crystals. It is a result of modulation in the electronic charge arising due to a periodic modulation in the crystal lattice. The 1T polymorph of another layered Tantalum di-chalcogenide, namely, $\mathrm{TaS}_{2}$, exhibits one of the strongest known CDW characteristics enabling temperature dependent

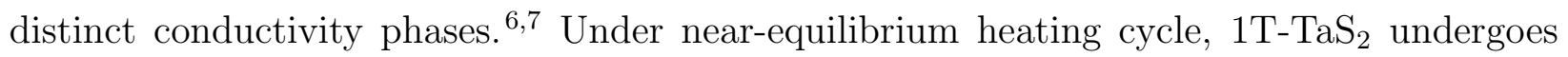
commensurate $(C)$ to triclinic $(T)$ phase transition at $223 \mathrm{~K}, T$ to nearly-commensurate $(N C)$ phase transition at $283 \mathrm{~K}, N C$ to incommensurate $(I C)$ phase transition at $353 \mathrm{~K}$ and $I C$ to normal metal beyond $550 \mathrm{~K} .{ }^{9}[1]$ When driven away from equilibrium condition, either by pulsed optical excitation or by an electric field, the crystal exhibits multiple metastable phases with different resistivities. $\frac{12}{14}$ In particular, the electrically driven phase transitions in $1 \mathrm{~T}-\mathrm{TaS}_{2}$, which result from joule heating, have attracted a lot of attention. $15+19$ This makes $1 \mathrm{~T}-\mathrm{TaS}_{2}$ a promising candidate for realizing various functionalities enabling metalbased electronics.

Negative differential resistance (NDR) is an important analog functionality widely used for a variety of applications including high-frequency microwave devices, local oscillators, amplifiers, switching circuits, and multi-valued logic. There have been several attempts of implementation of NDR using two-dimensional layered materials through Esaki diodes $20+24$ and resonant tunneling diodes $(\mathrm{RTD})^{25 \mid 27}$ by exploiting the atomically abrupt junctions - 
an important requirement to achieve high tunneling efficiency. In both cases, the carriers tunnel through a vertical junction between the valence band and the conduction band (for Esaki diode) or through a tunnel barrier (for RTD). Since both these working principles involve tunneling of carriers through a junction, the peak current density suffers significantly since it is difficult to achieve high tunneling efficiency in two-dimensional layered materials due to (a) relatively large effective mass, particularly in the out-of-plane direction, and (b) misalignment of crystals in vertical heterojunctions resulting in momentum mismatch. For Esaki diodes, a broken gap van der Waals heterojunction has been used recently to mitigate this. $\stackrel{28}{28}$ Nonetheless, low peak current density remains a key challenge in the layered material based NDR devices and is undesirable for many applications.

In this work, by exploiting electrically driven phase transitions in $1 \mathrm{~T}-\mathrm{TaS}_{2}$, we demonstrate a T-shaped asymmetric $1 \mathrm{~T}-\mathrm{TaS}_{2} / 2 \mathrm{H}-\mathrm{MoS}_{2}$ heterojunction based negative differential

resistor with a high peak current density $\left(J_{p}\right)$ among other layered material based NDR implementations. We are also able to effectively tune the peak current $\left(I_{p}\right)$ through both electrical and optical gating. $\mathrm{TaS}_{2}$ acts as an active material in the device and the working principle relies on the electrically driven phase transition in $\mathrm{TaS}_{2}$. On the other hand, the semiconducting $\mathrm{MoS}_{2}$ component in the device adds the gate voltage and light tunability in the obtained $I_{p}$. The mechanism does not depend on band-to-band tunneling effect and is extremely robust against interface quality, ambiance, and time induced degradation.

\section{Results and discussion}

Figure 1(a) schematically depicts the proposed device structure which is an asymmetric T-junction of multi-layer $1 \mathrm{~T}-\mathrm{TaS}_{2}$ and multi-layer $2 \mathrm{H}-\mathrm{MoS}_{2}$. To fabricate the T-junction devices, multi-layer $\mathrm{MoS}_{2}$ is first transferred on a doped silicon substrate covered by $285 \mathrm{~nm}$ thick thermally grown $\mathrm{SiO}_{2}$. This is followed by a transfer of the multi-layer $\mathrm{TaS}_{2}$ flake such that there is a strong asymmetry in length across the two arms of $\mathrm{TaS}_{2}$. The asymmetry 
and orientation between the two flakes are controlled by a combination of rotational and translational stages during the transfer process. Ni/Au electrodes are then patterned and deposited using the standard nano-fabrication process. The details of the device fabrication are provided in Methods section. An optical image and the thickness profile of a T-junction (device $\mathrm{D}_{1}$ ) after complete fabrication is shown in figure $1(\mathrm{~b})$, where $L_{1}$ and $L_{2}\left(L_{1}<L_{2}\right)$ are respectively the lengths of the top and bottom $\mathrm{TaS}_{2}$ arms. Figure 1(c)-(d) show the current-voltage characteristics of the heterojunction $\left(\mathrm{T}_{1} \mathrm{M}\right)$ probed between terminals $\mathrm{T}_{1}$ and $\mathrm{M}$ (with terminal $\mathrm{T}_{2}$ kept open) for different values of back-gate voltage $\left(V_{g}\right)$ at $290 \mathrm{~K}$ and $180 \mathrm{~K}$, respectively. The obtained on/off ratio is $\sim 2.8 \times 10^{4}$ at $290 \mathrm{~K}$ and $\sim 2.4 \times 10^{5}$ at $180 \mathrm{~K}$ with $V_{d}=-1 V$. Note that with $V_{T_{1}}>0$, Ni contact acts as the source and injects electrons into the $\mathrm{MoS}_{2}$ channel, as explained in the top right inset of figure 1(c). On the other hand, with $V_{T_{1}}<0$, it is the $\mathrm{TaS}_{2}$ that acts as the source and injects electrons into the $\mathrm{MoS}_{2}$ channel (top left inset). The drive current does not vary significantly for positive and negative $V_{T_{1}}$, suggesting similar carrier injection efficiency of $\mathrm{TaS}_{2}$ and Ni contacts to $\mathrm{MoS}_{2} \cdot{ }^{29}$ The result emphasizes the possible application of $\mathrm{TaS}_{2}$ as an efficient van der Waals contact which can maintain the pristine quality of the underneath layered semiconductor. Also, the traces exhibit no hysteresis between the forward and the reverse sweeps at both temperatures, suggesting traps do not play a significant role in these measurements. In analogue to $\mathrm{T}_{1} \mathrm{M}$, the current-voltage characteristics of $\mathrm{T}_{2} \mathrm{M}$ heterojunction (probed between terminals $\mathrm{T}_{2}$ and $\mathrm{M}$ with terminal $\mathrm{T}_{1}$ kept open) at $290 \mathrm{~K}$ and $180 \mathrm{~K}$ are outlined in Supporting Figure S1, which show characteristics similar to the $\mathrm{T}_{1} \mathrm{M}$ case.

Phase transition induced resistance switching in $1 \mathrm{~T}-\mathrm{TaS}_{2}$ has been extensively studied.13[15]19 The nearly-commensurate $(N C)$ to incommensurate $(I C)$ phase transition that normally occurs at $353 \mathrm{~K}$ under low electric field condition (quasi-equilibrium) can be electrically driven while operating at room temperature through joule heating. Figure 1(e) shows the current-voltage characteristics of the two-terminal $\mathrm{TaS}_{2}$ device (by probing terminals $\mathrm{T}_{1}$ and $\mathrm{T}_{2}$ of device $\mathrm{D}_{1}$ ), delineating the $N C$ - $I C$ phase transition at $1.8 \mathrm{~V}$. As we increase 
the voltage bias across $\mathrm{TaS}_{2}$ terminals, current-voltage characteristics deviate from linear to non-linear regime beyond $\sim 1 \mathrm{~V}$. With further increase in the voltage, the current increases abruptly by a factor of 1.56 which is in accordance with the change in the resistance during $N C$-IC phase transition. 13130131 This is further evident from the simulation outlined in Methods and results in Supporting Figure S2 that the joule heating induced increment in temperature in the channel is sufficient to result in $N C$ - $I C$ phase transition.

Gate tunable NDR and role of asymmetry: Exploiting the external bias driven phase transition in $1 \mathrm{~T}-\mathrm{TaS}_{2}$, we achieve a gate tunable NDR in the $1 \mathrm{~T}-\mathrm{TaS}_{2} / 2 \mathrm{H}-\mathrm{MoS}_{2}$ asymmetric $\mathrm{T}$-junction. The equivalent circuit of the 4-terminal device $\mathrm{D}_{1}$ embedded with crystal structure of $2 \mathrm{H}-\mathrm{MoS}_{2}$ as well as $1 \mathrm{~T}-\mathrm{TaS}_{2}$ in $N C$ and $I C$ phase is schematically represented in figure 2(a) and 2(b) respectively, wherein $R_{1}$ and $R_{2}$ correspond to the TaS $\mathrm{S}_{2}$ resistance in the arms above and below the junction region, and $R_{M}$ corresponds to the $\mathrm{MoS}_{2}$ resistance. The fourth terminal is connected to the back gate. The idea is to drive a high electrical field across the $\mathrm{TaS}_{2}$ terminals $\mathrm{T}_{1}$ and $\mathrm{T}_{2}$, thereby inducing the $N C$ - $I C$ phase transition that in turn reduces the resistance of the $\mathrm{TaS}_{2}$ branches, and simultaneously monitor the current through the $\mathrm{MoS}_{2}$ branch $\left(I_{M}\right)$. As the voltage $V_{T_{1}}$ increases and the $N C$ - $I C$ phase transition occurs, the current through $R_{2}$ increases abruptly which causes an abrupt reduction in $I_{M}$, thereby enabling NDR in the $\mathrm{MoS}_{2}$ branch.

If $n_{1}$ and $n_{2}$ are respectively the factors by which the resistance in $\mathrm{TaS}_{2}$ changes in the upper $\left(R_{1}\right)$ and the lower branches $\left(R_{2}\right)$ due to the $N C$-IC phase transition, the ratio $(\eta)$ of $I_{M}$ before and after the phase transition can be obtained using Kirchoff's law

$$
\eta=\frac{\left.I_{M}\right|_{\text {before transition }}}{\left.I_{M}\right|_{\text {after transition }}} \approx \frac{1+\left(\frac{n_{2}}{n_{1}}\right) \frac{R_{1}}{R_{2}}}{1+\frac{R_{1}}{R_{2}}}
$$

In Equation 1, we have used $R_{M} \gg R_{1}, R_{2}$ as observed from figure $1(\mathrm{c})$ and $1(\mathrm{e})$. One immediately realizes from the equation that it is important to maintain the asymmetry in 
the $\mathrm{TaS}_{2}$ branches to obtain NDR characteristics (that is, $\eta>1$ ), and a perfectly symmetric structure would result in $\eta=1$ and thus cannot cause NDR.

Figure 2(c) shows the gate voltage dependent NDR characteristics from device $\mathrm{D}_{1}$ for $V_{T_{1}}>0$ (in blue traces) and $V_{T_{1}}<0$ (in red traces). The corresponding PVCR values are plotted in figure 2(d). For $V_{T_{1}}>0$, the electrons flow from $\mathrm{MoS}_{2}$ to $\mathrm{TaS}_{2}$ at the junction without any appreciable energy barrier. Owing to the excellent gate modulation of the $\mathrm{MoS}_{2}$ channel resistance, we observe a strong gate voltage dependence of the peak current (region $\mathrm{A}$ and B), and $I_{p}$ reaches as high as $4 \mu \mathrm{A} \mu \mathrm{m}^{-1}$ at $V_{g}=50 \mathrm{~V}$ from $0.04 \mu \mathrm{A} \mu \mathrm{m}^{-1}$ at $V_{g}=-60 \mathrm{~V}$ as shown in figure 2(e) (filled blue spheres). The peak current density of the NDR device can be defined as $J_{p}=I_{p} /\left(W \times L_{T}\right)$ where $W$ and $L_{T}$ denote the width of the channel and transfer length of the $\mathrm{MoS}_{2} / \mathrm{TaS}_{2}$ contact respectively, as explained in figure 2(a)-(b). We obtain a high peak current density of $4.15 \times 10^{4} \mathrm{nA} \mu \mathrm{m}^{-2}$ from device $\mathrm{D}_{1}$ at room temperature which is superior to typical layered material based NDR devices. The device $\mathrm{D}_{1}$ exhibits a PVCR of about 1.06. Interestingly, the PVCR shows a very weak dependence on $V_{g}$ (top-filled blue pentagon in figure $2(\mathrm{~d})$ ), which is in good agreement with Equation 1, as $\eta$ is independent of $V_{g}$.

In case of $V_{T_{1}}<0$ (the red traces), that is when $\mathrm{TaS}_{2}$ injects electrons into $\mathrm{MoS}_{2}$, the electrons need to overcome the $\mathrm{TaS}_{2} / \mathrm{MoS}_{2}$ Schottky barrier. It has been recently demonstrated that during an $N C$-IC phase transition, the change in $\mathrm{TaS}_{2}$ resistance is accompanied by a suppression in the Schottky barrier height $(\mathrm{SBH})$ at the $\mathrm{TaS}_{2} / \mathrm{MoS}_{2}$ junction. $\frac{29}{29}$ These two effects compete with each other in the final device characteristics since a suppression in the $\mathrm{SBH}$ would result in an abrupt enhancement in the $I_{M}$, rather than NDR. For $V_{g} \leq-10$ V (region C), the barrier height suppression plays the dominant role, and thus instead of NDR, we observe an increment in the current $I_{M}$, as indicated by a PVCR of $<1$ in figure 2(d). However, with an increase in $V_{g}$ (region D), the electrons can tunnel through the $\mathrm{TaS}_{2} / \mathrm{MoS}_{2}$ Schottky barrier due to strong band bending (see figure 2(f)), hence the barrier height suppression does not play a significant role, and the NDR characteristics reappear. 
The simultaneous modulation of the barrier height at the $\mathrm{TaS}_{2} / \mathrm{MoS}_{2}$ junction along with the resistivity switching during $N C-I C$ phase transition provides an additional tunability in the NDR device when $V_{T_{1}}<0$.

Now, if we apply the bias at terminal $\mathrm{T}_{2}$ while connecting $T_{1}$ and $\mathrm{M}$ to a common ground, then the NDR gets completely suppressed for both $V_{T_{2}}>0$ and $V_{T_{2}}<0$, even for large positive gate voltage (see Supporting Figure S3). This can be easily explained using Equation 11, keeping in mind that $R_{1}$ and $R_{2}$ are now exchanged. The second term in both numerator and denominator are now small compared to 1 , leading to $\eta \approx 1$, which suppresses any NDR.

Note that the $N C$-IC phase transition is accompanied by hysteresis (see, for example, figure 1(e)) which manifests as a hysteresis window in the NDR characteristics as well. The hysteresis window can be reduced by introducing fast heat dissipation channels in the device. We fabricate a second device $\left(\mathrm{D}_{2}\right)$ where the $\mathrm{MoS}_{2}$ layer underneath is a wide one, facilitating improved heat dissipation, as explained in Supporting Figure S4. The external bias dependent current-voltage characteristics of the $\mathrm{TaS}_{2}$ sample exhibit negligible hysteresis. This manifests in the reduced hysteresis window when the device is operated in the NDR mode, both for $V_{T_{1}}>0$ and $V_{T_{1}}<0$. Note that the two $\mathrm{TaS}_{2}$ branches are more symmetric in $\mathrm{D}_{2}$ than in $\mathrm{D}_{1}$, which results in a slightly reduced value of the PVCR, further verifying the operation principle described earlier.

Light tunable NDR: Before demonstrating the light tunable NDR effect, we first show the efficient phototransistor effect by light gating achieved in the heterojunction. The configuration is shown in figure 3a. Figure $3 \mathrm{~b}$ shows the photo-induced current-voltage characteristics of the heterojunction $\mathrm{T}_{1} \mathrm{M}$ (probed between terminal $\mathrm{T}_{1}$ and $\mathrm{M}$ with terminal $\mathrm{T}_{2}$ kept open) at $V_{g}=-50 \mathrm{~V}$ (See Supporting Figure S5 for characteristics at other gate voltage values). When excited with a laser of wavelength $532 \mathrm{~nm}$ and a spot size encompassing the entire device, the photocurrent across the device can be effectively modulated with the excitation 
power. In particular, when the device is turned off by applying $V_{g}=-60 \mathrm{~V}$, with an excitation power $\left(P_{o p}\right)$ of $45.4 \mathrm{nW}$, the device current switches by a factor of $1.65 \times 10^{2}$ for $V_{T_{1}}=1 \mathrm{~V}$, and $2.52 \times 10^{3}$ for $V_{T_{1}}=-1 \mathrm{~V}$ from the dark condition. The responsivity of the device is extracted as $R=\frac{I_{\text {light }}-I_{\text {dark }}}{P_{\text {op }}}$ where $I_{\text {light }}$ and $I_{\text {dark }}$ are the current with and without light, respectively. The calculated $R$ is plotted as a function of incident optical power for $V_{T_{1}}=-1 \mathrm{~V}$ in figure $3(\mathrm{c}) . R$ increases with a reduction in the excitation power, and reaches a value as high as $2.31 \times 10^{4} A W^{-1}$ at an excitation power of $0.22 \mathrm{nW}$.

We now exploit this photo-induced gating effect in the 4-terminal measurement to demonstrate light tunable NDR. As before, during this measurement, bias is applied in the terminal $\mathrm{T}_{1}$ for different values of the back gate voltage, keeping terminals $\mathrm{T}_{2}$ and $\mathrm{M}$ grounded. The results are shown in figure $3(\mathrm{~d})$-(f) for $V_{g}=-50,-20$, and $10 \mathrm{~V}$ with $V_{T_{1}}>0 \mathrm{~V}$. We observe that the NDR characteristics can be effectively tuned by the excitation power owing to a photogating induced modulation in the resistance of the $\mathrm{MoS}_{2}$ branch with optical power. Given the low power of the incident optical excitation, we do not expect any modulation of $\mathrm{TaS}_{2}$ conductivity with light. The simultaneous control of $I_{p}$ by $V_{g}$ and $P_{o p}$ is depicted in the color plot in figure 3g, which suggests that the degree of photo-tunability of the NDR is a strong function of the applied back gate voltage. Even when $V_{g}=-50 \mathrm{~V}$, with photoexcitation, we are able to achieve a peak current which is in excess of $1 \mu \mathrm{A} \mu \mathrm{m}^{-1}$, with a PVCR of $\sim 1.065$. Such a light and gate voltage tunable NDR characteristics are promising for light-gated optoelectronic applications. For $V_{T_{1}}<0 \mathrm{~V}$, where $\mathrm{TaS}_{2}$ injects electrons to $\mathrm{MoS}_{2}$ over an SBH, we obtain an optical power-dependent enhancement in the current rather than NDR effect at the phase transition, as explained in Supporting Figure S6. This is in agreement with the phase transition induced $\mathrm{TaS}_{2} / \mathrm{MoS}_{2}$ barrier suppression effect discussed earlier.

Enhancing the PVCR: An enhanced switching ratio during the $\mathrm{TaS}_{2}$ phase transition is desirable to further improve the PVCR in the proposed T-junction. In order to do so, we 
exploit electrically driven phase transition in $\mathrm{TaS}_{2}$ at low temperature. $\frac{13|16| 19}{1 n}$ addition to the equilibrium states, $1 \mathrm{~T}-\mathrm{TaS}_{2}$ exhibits several metastable states that can be accessed by electric field ${ }^{13}$ and optical pulse excitation. ${ }^{12[14}$ Figure 4(a) shows the current-voltage characteristics when bias is applied across the two terminals of $\mathrm{TaS}_{2}\left(\mathrm{~T}_{1}\right.$ and $\left.\mathrm{T}_{2}\right)$ at $160 \mathrm{~K}, 170$ $\mathrm{K}$ and $180 \mathrm{~K}$. The base sample temperature is intentionally kept below the $C$ - $T$ transition temperature to electrically drive the system into metastable state and eventually drive to $I C$ state, which causes an abrupt increase in the current by a factor of $\sim 2.23$.

In the corresponding four-terminal measurement at $180 \mathrm{~K}, I_{M}$ is plotted as a function $V_{T_{1}}$ in figure $4(\mathrm{~b})$. For $V_{T_{1}}>0$, the peak current shows a strong modulation by the gate voltage. The obtained PVCR is plotted in figure $4(\mathrm{c})$ as a function of $V_{g}$, and is found to show a weak $V_{g}$ dependence as expected from Equation 1, and saturates around 1.2, which is an improvement over the value obtained at room temperature. In contrast to figure $2(\mathrm{~d})$, the peak-to-valley transition at low temperature depicts multiple transitions (see top right inset of figure $4 \mathrm{~b}$ ), which results from the existence of the multiple metastable states in $1 \mathrm{~T}-\mathrm{TaS}_{2}$ under non-equilibrium.

For $V_{T_{1}}<0$, similar to figure $2(\mathrm{~d})$, we obtain two distinct regions of operation. When $V_{g} \leq-10 \mathrm{~V}$, we obtain a strong enhancement in $I_{M}$ due to the phase transition, and enhancement factor increases for larger negative $V_{g}$. In the left inset of figure 4(b), we show $I_{M}$ in log scale, delineating the abrupt increment in the current, which is enhanced compared with what we obtained at room temperature in figure 2(c). The stronger enhancement in $I_{M}$ and its gate voltage dependence is a clear indication of a strong suppression of the SBH at the $\mathrm{TaS}_{2}-\mathrm{MoS}_{2}$ interface during the meta-stable state to $I C$ phase transition. On the other hand, when $V_{g} \geq 10 \mathrm{~V}$, the electrons tunnel through the Schottky barrier due to strong band bending and the barrier suppression effect plays a less significant role. Under this condition, the NDR effect reappears due to the phase transition induced resistance switching of $\mathrm{TaS}_{2}$.

There is also a third regime of operation for $V_{T_{1}}<0$ around $V_{g}=0 \mathrm{~V}$, where we obtain a high PVCR of 1.59 (figure 4(c)) which cannot be explained just by the phase transition 
induced current division effect. This region of operation is completely absent for $V_{T_{1}}>0$. We also note a hysteresis associated with the current-voltage characteristics for negative $V_{T_{1}}$. This suggests the role of carrier trapping at the $\mathrm{TaS}_{2} / \mathrm{MoS}_{2}$ junction in the increased PVCR. During the $\mathrm{TaS}_{2}$ phase transition, the local temperature at the junction increases abruptly due to a sudden increase in the current $I_{T_{1}}$. This is supported by the simulation predicted temperature at the junction during the voltage sweep, as shown in figure 4(d). This results in activated local trapping of carriers, which in turn causes a screening of the gate field, reducing the magnitude of $I_{M}$. Note that with an increment in the gate voltage to higher positive values, the carriers tunnel through this barrier, and thus such trapping effects do not play a significant role.

The above discussion on the enhancement of PVCR using CDW phase transition at low temperature provides key insights about the device functionality, and has important implication on several niche applications (for example, space electronics) where both low temperature operation and radiation hardness are necessary. However, the improved performance at low temperature may have limited impact on applications requiring room temperature operation. In order to increase the PVCR at room temperature, the upper $\mathrm{TaS}_{2}$ branch $\left(R_{1}\right)$ of the T-network in Figure $2 \mathrm{a}-\mathrm{b}$ can be replaced by a constant resistance where no such phase transition occurs. This forces $n_{1}$ in Equation 1 to unity, which thus enhances $\eta$ and hence, the PVCR. In such a design, the PVCR will have an upper limit of $n_{2}$ with a suitable design ensuring $R_{1}>>R_{2}$.

While the additional terminals in the proposed multi-terminal device provide gate control and access to more state variables, from an implementation point of view, it would require some changes in the conventional NDR-based circuit design. For example, to implement an oscillator, where the positive resistance of a tank circuit is compensated by the negative resistance, the top $\left(\mathrm{T}_{1}\right)$ and right $(\mathrm{M})$ ports (see Figure $2 \mathrm{a}-\mathrm{b}$ ) can be directly used, grounding terminal $\mathrm{T}_{2}$.

The heterojunctions studied here are robust against ambience induced degradation and 
exhibit good repeatability of the data measured over more than a month (see Supporting Figure S7).In Table 1 we benchmark the performance of the devices reported here against other NDR devices based on both layered materials and bulk semiconductors. The peak current density of the proposed device exceeds the numbers reported from layered materials based tunneling devices ${ }^{20 \mid}$, 28 , and also compares well with several bulk technologies ${ }^{32}-37$. With further optimization, the proposed device is promising to be comparable with SiGe and III-V semiconductor based complex multi-heterojunctions with highest reported peak current densities $\sqrt{38}[40$.

Active metal based electronics, such as the current design, is expected to provide radiation hardness. In a conventional tunnel diode, a major cause for performance degradation due to exposure to radiation is an increase in the excess current, and in turn a degradation in the PVCR ${ }^{41}$. This is primarily due to a degradation in the tunnel junction interface due to

defect generation, resulting in an increase in the trap assisted tunneling ${ }^{42}$. The proposed device is expected to have an advantage in this aspect since it avoids any tunnel junction. Equation 1 suggests that the PVCR in the device only depends on the resistance components (and switching ratios) of the $\mathrm{TaS}_{2}$ branches, and independent of the semiconducting $\mathrm{MoS}_{2}$ component, and thus the PVCR is inherently radiation hard. Finally, one could, in principle, replace the semiconducting $\mathrm{MoS}_{2}$ component by a metallic branch of appropriate resistance to make it more radiation hard. The PVCR can still be maintained based on Equation 1. though the optical control or the electrical gate control offered by the semiconducting component will be lost.

\section{Conclusion:}

In conclusion, we demonstrated a different way of achieving negative differential resistance by simultaneously exploiting the resistivity and Schottky barrier height switching using an electrically driven phase transition arising from charge density wave in a $1 \mathrm{~T}-\mathrm{TaS}_{2} / 2 \mathrm{H}-\mathrm{MoS}_{2}$ 
heterojunction. The negative differential resistance can be effectively tuned by external stimuli including photo-excitation and a back gate voltage. The design of the device distinguishes itself from other tunneling based NDR implementations by using majority carriers and not requiring any stringent interface quality - thus achieving a high peak current density. The proposed device also exhibits excellent air stability and repeatability. The results are promising towards the implementation of various functionalities employing robust and tunable negative differential resistance, and mark a step towards $1 \mathrm{~T}-\mathrm{TaS}_{2}$ based metal electronics.

\section{Methods}

T-junction device fabrication and characterization: The bulk crystals of $1 \mathrm{~T}-\mathrm{TaS}_{2}$ and $2 \mathrm{H}-\mathrm{MoS}_{2}$ are procured from the 2D Semiconductors. $1 \mathrm{~T}-\mathrm{TaS}_{2} / 2 \mathrm{H}-\mathrm{MoS}_{2}$ T-junction is fabricated in two steps: first, the thin $\mathrm{MoS}_{2}$ flakes are mechanically exfoliated from scotch tape onto a heavily doped Si substrate coated with $285 \mathrm{~nm}$ thick $\mathrm{SiO}_{2}$ using polydimethylsiloxane (PDMS). Second, the $\mathrm{TaS}_{2}$ flakes are transferred through PDMS attached to a glass slide that is controlled by an additional manipulator. The alignment of the $\mathrm{TaS}_{2}$ flake with respect to the $\mathrm{MoS}_{2}$ flake is controlled by the rotational stage in order to form the T-junction. The whole exfoliation and transfer processes are done at room temperature, with no additional cleaning step. The substrate is then spin coated with a high contrast positive resist - polymethyl methacrylate (PMMA) $950 \mathrm{C} 3$ and softly baked for 2 minutes at $180{ }^{\circ} \mathrm{C}$ for pattern writing by means of electron beam lithography with an electron beam dose of 200 $\mu \mathrm{C} \mathrm{cm}{ }^{-2}$, an electron beam current of $300 \mathrm{pA}$, and an acceleration voltage of $20 \mathrm{KV}$. The pattern development is carried out in $1: 3 \mathrm{MIBK} / \mathrm{IPA}$ developer solution followed by IPA wash and blow drying in $\mathrm{N}_{2}$. Metal contacts are formed by blanket deposition of $10 \mathrm{~nm}$ $\mathrm{Ni} / 50 \mathrm{~nm}$ Au using a DC magnetron sputter coating system in presence of Ar plasma at $6.5 \times 10^{-3}$ Torr. Excess metal lift-off is carried out by immersing the substrate in acetone 
for 15 - 30 minutes followed by IPA wash for 30 seconds and blow drying in $\mathrm{N}_{2}$. Buffered HF solution is used to etch the back oxide from the substrate and highly conducting silver paste is used for the back gate contact.

The electrical measurements are performed in a probe station with a base vacuum level of about $3.45 \times 10^{-3}$ Torr at room temperature. The low temperature measurements are performed with liquid $\mathrm{N}_{2}$ supply at a base vacuum of $6.45 \times 10^{-6}$ Torr. Measurements under light illumination are performed using a $532 \mathrm{~nm}$ laser, with the spot size being larger than the device area. The optical power is calibrated for the area factor and the loss due to the transmission through the optical window of the probe station.

Thermal model Simulation: The temperature variation along the $\mathrm{TaS}_{2}$ channel resulting from joule heating is obtained by solving the one-dimensional heat equation described below using finite element method: 19143144

$$
A \frac{d}{d x}\left(K \frac{d T}{d x}\right)+P=g\left(T-T_{\text {base }}\right)
$$

where

$P \quad=$ Heat generation rate per unit channel length.

$A=$ Cross-sectional area of the device.

$K=$ Thermal conductivity of $1 \mathrm{~T}-\mathrm{TaS}_{2}$.

$T_{\text {base }}=$ Base temperature.

$g \quad=$ Thermal conductance of the substrate per unit channel length.

This results in a non-uniform rise in temperature along the channel. We incorporate the temperature dependence of the thermal conductivity $(K)^{45}$ in the model. At high bias, the temperature increases abruptly in the middle of the channel $(x=4.68 \mu m)$ during the phase transition as shown in figure S2(a) and (c) for $298 \mathrm{~K}$ and $180 \mathrm{~K}$ respectively. The simulated temperature profile across the $\mathrm{TaS}_{2}$ channel shows strong non-uniformity for a base temperature of $298 \mathrm{~K}$ and $180 \mathrm{~K}$ at different biasing conditions (see figure (b) and 
(d) of Supporting Figure S2). The temperature drops near the ends of the channel due to the heat sink nature of $\mathrm{Ni} / \mathrm{Au}$ contact. However, the temperature in the middle of the channel increases with the external bias which confirms the non-uniformity of the Joule heating induced temperature change. Owing to the non-equilibrium heat generation in the $\mathrm{TaS}_{2}$ channel, the phase transitions are achieved through multiple metastable states.

\section{Acknowledgement}

K.M. acknowledges the grant from Indian Space Research Organization (ISRO), grant under Ramanujan Fellowship, the Early Career Award, and Nano Mission from the Department of Science and Technology (DST), and support from MHRD, MeitY and DST Nano Mission through NNetRA.

\section{Associated Content}

The authors declare no competing financial or non-financial Interests.

\section{Supporting Information Available}

The following files are available free of charge. Supporting information available on the following: Electrical Characterization of $\mathrm{TaS}_{2} / \mathrm{MoS}_{2}$ junction; Simulated temperature variation along $\mathrm{TaS}_{2}$ channel; Characteristics for $\mathrm{T}_{2}$ biasing in device $\mathrm{D}_{1}$; Gate tunable NDR with reduced hysteresis in $1 \mathrm{~T}-\mathrm{TaS}_{2} / 2 \mathrm{H}-\mathrm{MoS}_{2}$ asymmetric T-junction device $\left(\mathrm{D}_{2}\right)$; Light response of heterojunction $\mathrm{T}_{1} \mathrm{M}$ of device $\mathrm{D}_{1}$; Light controlled $\mathrm{NDR}$ in $\mathrm{TaS}_{2} / \mathrm{MoS}_{2}$ asymmetric T-junction $\left(\mathrm{D}_{1}\right)$; Stability of $\mathrm{TaS}_{2} / \mathrm{MoS}_{2}$ asymmetric T-junction $\left(\mathrm{D}_{1}\right)$. 


\section{References}

1. Zhang, C.; Wang, B.; Duan, G.; Zhang, E.; Fleetwood, D.; Alles, M.; Schrimpf, R.; Rooney, A.; Khestanova, E.; Auton, G.; Gorbachev, R.; Haigh, S.; Pantelides, S. Total Ionizing Dose Effects on hBN Encapsulated Graphene Devices. IEEE Trans. Nucl. Sci. 2014, 61, 2868-2873.

2. Gao, L.; Holbert, K. E.; Yu, S. Total Ionizing Dose Effects of Gamma-Ray Radiation on NbOx-Based Selector Devices for Crossbar Array Memory. IEEE Trans. Nucl. Sci. 2017, 64, 1535-1539.

3. Liu, G.; Zhang, E.; Bloodgood, M.; Liang, C.; Salguero, T.; Fleetwood, D.; Balandin, A. Total Ionizing Dose Effects on Threshold Switching in 1T-TaS ${ }_{2}$ Charge Density Wave Devices. IEEE Electron Device Lett. 2017, 38, 1724-1727.

4. Geremew, A.; Kargar, F.; Zhang, E.; Zhao, S.; Aytan, E.; Bloodgood, M.; Salguero, T.; Rumyantsev, S.; Fedoseyev, A.; Fleetwood, D.; Balandin, A. Proton-IrradiationImmune Electronics Implemented with Two-Dimensional Charge-Density-Wave Devices. Nanoscale 2019, 11, 8380-8386.

5. Khitun, A.; Geremew, A. K.; Balandin, A. A. Transistor-Less Logic Circuits Implemented with 2-D Charge Density Wave Devices. IEEE Electron Device Lett. 2018, 39, $1449-1452$.

6. Wilson, J.; Salvo, F. D.; Mahajan, S. Charge-Density Waves and Superlattices in the Metallic Layered Transition Metal Dichalcogenides. Adv. Phys. 1975, 24, 117-201.

7. Williams, P.; Scruby, C.; Clark, W.; Parry, G. Charge Density Waves in the Layered Transition Metal Dichalcogenides. J. Phys., Colloq. 1976, 37, C4-139.

8. Mahajan, M.; Kallatt, S.; Dandu, M.; Sharma, N.; Gupta, S.; Majumdar, K. Light 
Emission from the Layered Metal 2H-TaSe 2 and its Potential Applications. Commun. Phys. 2019, 2, 1-9.

9. Manzke, R.; Buslaps, T.; Pfalzgraf, B.; Skibowski, M.; Anderson, O. On the Phase Transitions in 1T-TaS 2 . Europhys. Lett. 1989, 8, 195-200.

10. Ishiguro, T.; Sato, H. Electron Microscopy of Phase Transformations in 1T-TaS 2 . Phys. Rev. B 1991, 44, 2046-2060.

11. Thomson, R. E.; Burk, B.; Zettl, A.; Clarke, J. Scanning Tunneling Microscopy of the Charge-Density-Wave Structure in $1 \mathrm{~T}-\mathrm{TaS}_{2}$. Phys. Rev. B 1994, 49, 16899-16916.

12. Stojchevska, L.; Vaskivvskyi, I.; Mertelj, T.; Svetin, D.; Brazovskii, S.; Mihailovic, D. Ultrafast Switching to a Stable Hidden Quantum State in an Electronic Crystal. Science (Washington, DC, U. S.) 2014, 344, 177-180.

13. Yoshida, M.; Suzuki, R.; Zhang, Y.; Nakano, M.; Iwasa, Y. Memristive Phase Switching in Two-Dimensional 1T-TaS 2 Crystals. Sci. Adv. 2015, 1, 1-7.

14. Vaskivskyi, I.; Gospodaric, J.; Brazovskii, S.; Svetin, D.; Sutar, P.; Goreshnik, E.; Mihailovic, I. A.; Mertelj, T.; Mihailovic, D. Controlling the Metal-To-Insulator Relaxation of the Metastable Hidden Quantum State in 1T-TaS ${ }_{2}$. Sci. Adv. 2015, 1, 1-6.

15. Hollander, M. J.; Liu, Y.; Lu, W. J.; Li, L. J.; Sun, Y. P.; Robinson, J. A.; Datta, S. Electrically Driven Reversible Insulator-Metal Phase Transition in 1T-TaS 2 . Nano Lett. 2015, 15, 1861-1866.

16. Liu, G.; Debnath, B.; Pope, T. R.; Salguero, T. T.; Lake, R. K.; Balandin, A. A. A Charge-Density-Wave Oscillator Based on an Integrated Tantalum Disulfide-Boron Nitride-Graphene Device Operating at Room Temperature. Nat. Nanotechnol. 2016, $11,845-850$. 
17. Yoshida, M.; Gokuden, T.; Suzuki, R.; Nakano, M.; Iwasa, Y. Current Switching of Electronic Structures in Two-Dimensional 1T-TaS ${ }_{2}$ Crystals. Phys. Rev. B 2017, 95, $1-5$.

18. Zheng, S.; Liu, F.; Zhu, C.; Liu, Z.; Fan, H. J. Room-Temperature Electrically Driven Phase Transition of Two-Dimensional 1T-TaS ${ }_{2}$ Layers. Nanoscale 2017, 9, 2436-2441.

19. Zhu, C.; Chen, Y.; Liu, F.; Zheng, S.; Li, X.; Chaturvedi, A.; Zhou, J.; Fu, Q.; He, Y.; Zeng, Q.; Fan, H. J.; Zhang, H.; Liu, W. J.; Yu, T.; Liu, Z. Light-Tunable 1T-TaS ${ }_{2}$ Charge-Density-Wave Oscillators. ACS Nano 2018, 12, 11203-11210.

20. Yan, R.; Fathipour, S.; Han, Y.; Song, B.; Xiao, S.; Li, M.; Ma, N.; Protasenko, V.; Muller, D. A.; Jena, D.; Xing, H. G. Esaki Diodes in van der Waals Heterojunctions with Broken-Gap Energy Band Alignment. Nano Lett. 2015, 15, 5791-5798.

21. Roy, T.; Tosun, M.; Cao, X.; Fang, H.; Lien, D. H.; Zhao, P.; Chen, Y. Z.; Chueh, Y. L.; Guo, J.; Javey, A. Dual-Gated $\mathrm{MoS}_{2} / \mathrm{WSe}_{2}$ van der Waals Tunnel Diodes and Transistors. ACS Nano 2015, 9, 2071-2079.

22. Roy, T.; Tosun, M.; Hettick, M.; Ahn, G. H.; Hu, C.; Javey, A. 2D-2D Tunneling FieldEffect Transistors Using $\mathrm{WSe}_{2} / \mathrm{SnSe}_{2}$ Heterostructures. Appl. Phys. Lett. 2016, 108.

23. Nourbakhsh, A.; Zubair, A.; Dresselhaus, M. S.; Palacios, T. Transport Properties of a $\mathrm{MoS}_{2} / \mathrm{WSe}_{2}$ Heterojunction Transistor and its Potential for Application. Nano Lett. 2016, 16, 1359-1366.

24. Fan, S.; Vu, Q. A.; Lee, S.; Phan, T. L.; Han, G.; Kim, Y.-M.; Yu, W. J.; Lee, Y. H. Tunable Negative Differential Resistance in van der Waals Heterostructures at Room Temperature by Tailoring the Interface. ACS Nano 2019, 13, 8193-8201.

25. Britnell, L.; Gorbachev, R. V.; Geim, A. K.; Ponomarenko, L. A.; Mishchenko, A.; Greenaway, M. T.; Fromhold, T. M.; Novoselov, K. S.; Eaves, L. Resonant Tunnelling 
and Negative Differential Conductance in Graphene Transistors. Nat. Commun. 2013, 4, 1794-1795.

26. Nguyen, L. N.; Lan, Y. W.; Chen, J. H.; Chang, T. R.; Zhong, Y. L.; Jeng, H. T.; Li, L. J.; Chen, C. D. Resonant Tunneling through Discrete Quantum States in Stacked Atomic-Layered $\mathrm{MoS}_{2}$. Nano Lett. 2014, 14, 2381-2386.

27. Lin, Y. C.; Ghosh, R. K.; Addou, R.; Lu, N.; Eichfeld, S. M.; Zhu, H.; Li, M. Y.; Peng, X.; Kim, M. J.; Li, L. J.; Wallace, R. M.; Datta, S.; Robinson, J. A. Atomically Thin Resonant Tunnel Diodes Built from Synthetic van der Waals Heterostructures. Nat. Commun. 2015, 6, 1-6.

28. Shim, J.; Oh, S.; Kang, D. H.; Jo, S. H.; Ali, M. H.; Choi, W. Y.; Heo, K.; Jeon, J.; Lee, S.; Kim, M.; Song, Y. J.; Park, J. H. Phosphorene/Rhenium Disulfide Heterojunction-Based Negative Differential Resistance Device for Multi-Valued Logic. Nat. Commun. 2016, 7, 1-8.

29. Mahajan, M.; Murali, K.; Kawatra, N.; Majumdar, K. Gate-Controlled Large Resistance Switching Driven by Charge-Density Wave in $1 \mathrm{~T}-\mathrm{TaS}_{2} / 2 \mathrm{H}-\mathrm{MoS}_{2}$ Heterojunctions. Phys. Rev. Appl. 2019, 11, 1-10.

30. Sipos, B.; Kusmartseva, A.; Akrap, A.; Berger, H.; Forr, L.; Tutis, E. From Mott State to Superconductivity in $1 \mathrm{~T}-\mathrm{TaS}_{2}$. Nat. Mater. 2008, 7, 960965.

31. Yu, Y.; Yang, F.; Lu, X. F.; Yan, Y. J.; Cho, Y.-H.; Ma, L.; Niu, X.; Kim, S.; Son, Y.-W.; Feng, D.; Li, S.; Cheong, S.; Chen, X.; Zhang, Y. Gate-Tunable Phase Transitions in Thin Flakes of 1T-TaS 2 . Nat. Nanotechnol. 2015, 10 3, 270-6.

32. Oehme, M.; Sarlija, M.; Hahnel, D.; Kaschel, M.; Werner, J.; Kasper, E.; Schulze, J. Very High Room-Temperature Peak-To-Valley Current Ratio in Si Esaki Tunneling Diodes (March 2010). IEEE Trans. Electron Devices 2010, 57, 2857-2863. 
33. Oehme, M.; Haehnel, D.; Werner, J.; Kaschel, M.; Kirfel, O.; Kasper, E.; Schulze, J. Si Esaki Diodes with High Peak to Valley Current Ratios. Appl. Phys. Lett. 2009, 95, 242109 .

34. Oehme, M.; Karmous, A.; Sarlija, M.; Werner, J.; Kasper, E.; Schulze, J. Ge Quantum Dot Tunneling Diode with Room Temperature Negative Differential Resistance. Appl. Phys. Lett. 2010, 97, 012101-012101.

35. Park, S.; Anisha, R.; Berger, P. R.; Loo, R.; Nguyen, N. D.; Takeuchi, S.; Caymax, M. $\mathrm{Si} /$ SiGe Resonant Interband Tunneling Diodes Incorporating $\delta$-Doping Layers Grown by Chemical Vapor Deposition. IEEE Electron Device Lett. 2009, 30, 1173-1175.

36. Duschl, R.; Eberl, K. Physics and Applications of Si/SiGe/Si Resonant Interband Tunneling Diodes. Thin Solid Films 2000, 380, 151-153.

37. Golka, S.; Pfluegl, C.; Schrenk, W.; Strasser, G.; Skierbiszewski, C.; Siekacz, M.; Grzegory, I.; Porowski, S. Negative Differential Resistance in Dislocation-Free GaN/AlGaN Double-Barrier Diodes Grown on Bulk GaN. Appl. Phys. Lett. 2006, 88, 172106.

38. Bayram, C.; Vashaei, Z. AlN/GaN Double-Barrier Resonant Tunneling Diodes Grown by Metal-Organic Chemical Vapor Deposition. Appl. Phys. Lett. 2010, 96.

39. Chung, S.-Y.; Yu, R.; Jin, N.; Park, S.-Y.; Berger, P.; Thompson, P. Si/SiGe Resonant Interband Tunnel Diode with $\mathrm{f}_{\mathrm{r} 0} 20.2 \mathrm{GHz}$ and Peak Current Density $218 \mathrm{kA} / \mathrm{cm}^{2}$ for K-Band Mixed-Signal Applications. IEEE Electron Device Lett. 2006, 27, 364 - 367.

40. Pawlik, D.; Romanczyk, B.; Thomas, P.; Rommel, S.; Edirisooriya, M.; ContrerasGuerrero, R.; Droopad, R.; Loh, W.; Wong, M. H.; Majumdar, K.; Wang, W. .; Kirsch, P. D.; Jammy, R. Benchmarking and Improving III-V Esaki Diode Performance with a Record 2.2 MA $/ \mathrm{cm}^{2}$ Peak Current Density to Enhance TFET Drive Current. 2012 International Electron Devices Meeting. 2012; pp 27.1.1-27.1.3. 
41. Soliman, F. A. S.; Kamh, S. A. Radiation Damage in Negative-Differential Resistance Devices. J. Mater. Sci.: Mater. Electron. 1994, 5, 30-34.

42. Majumdar, K.; Thomas, P.; Loh, W.; Hung, P.; Matthews, K.; Pawlik, D.; Romanczyk, B.; Filmer, M.; Gaur, A.; Droopad, R.; Rommel, S. L.; Hobbs, C.; Kirsch, P. D. Mapping Defect Density in MBE Grown $\operatorname{In}_{0.53} \mathrm{Ga}_{0.47}$ As Epitaxial Layers on Si Substrate Using Esaki Diode Valley Characteristics. IEEE Trans. Electron Devices 2014, 61, 2049 2055.

43. Grosse, K. L.; Bae, M.-H.; Lian, F.; Pop, E.; King, W. P. Nanoscale Joule Heating, Peltier Cooling and Current Crowding at Graphene-Metal Contacts. Nat. Nanotechnol. 2011, 6 5, 287-90.

44. Geremew, A. K.; Rumyantsev, S.; Kargar, F.; Debnath, B.; Nosek, A.; Bloodgood, M. A.; Bockrath, M.; Salguero, T. T.; Lake, R. K.; Balandin, A. A. Bias-Voltage Driven Switching of the Charge-Density-Wave and Normal Metallic Phases in 1T-TaS ${ }_{2}$ Thin-Film Devices. ACS Nano 2019, 13, 7231-7240.

45. Núñez Regueiro, M. D.; Lopez-Castillo, J. M.; Ayache, C. Thermal Conductivity of $1 T-\mathrm{TaS}_{2}$ and $2 \mathrm{H}-\mathrm{TaSe}_{2}$. Phys. Rev. Lett. 1985, 55, 1931-1934. 

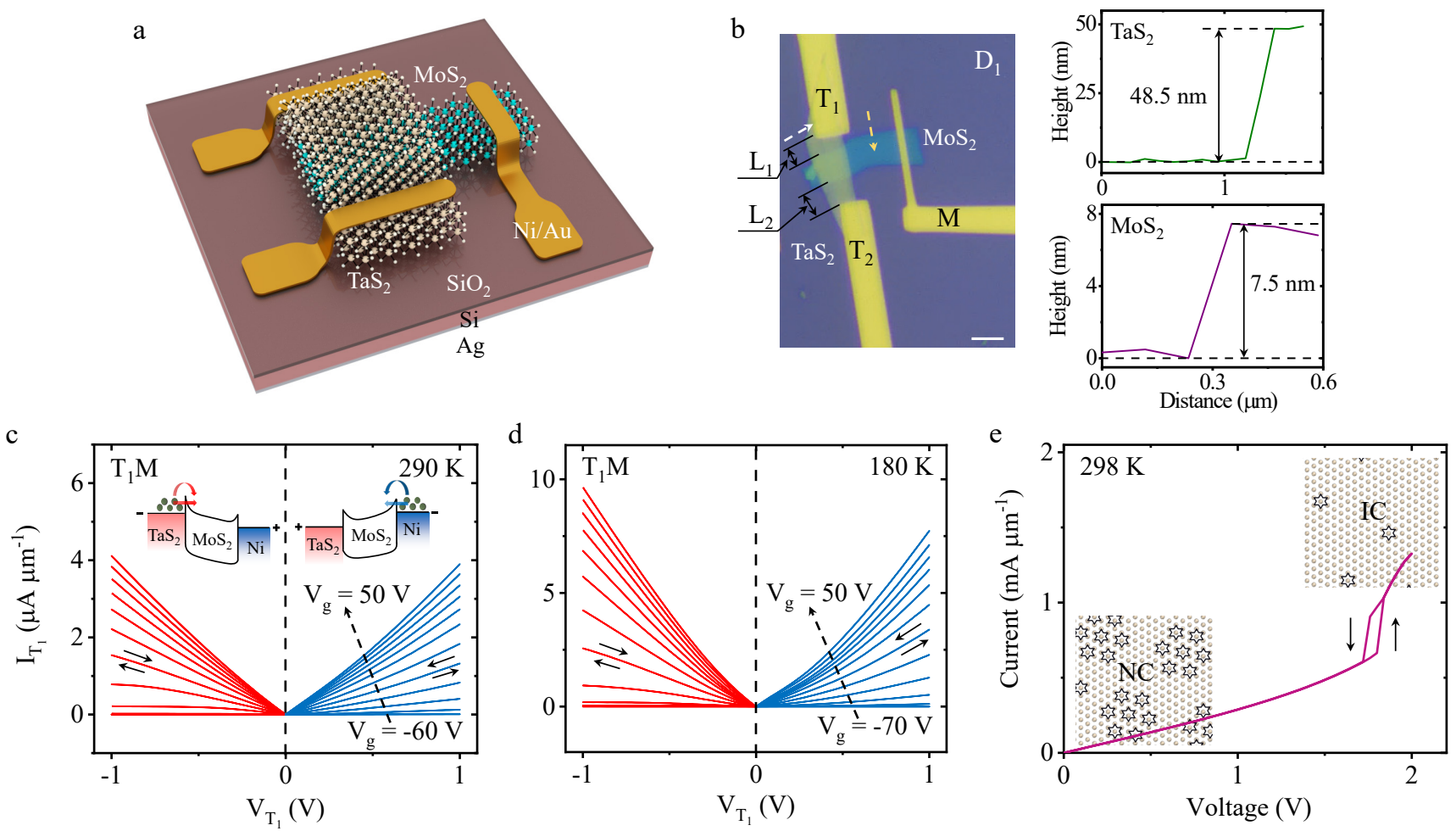

Figure 1: Electrical Characterization of $1 \mathrm{~T}-\mathrm{TaS}_{2} / 2 \mathrm{H}-\mathrm{MoS}_{2}$ asymmetric Tjunction. (a) Schematic representation of the T-Junction. (b) Left panel: Optical image of the fabricated asymmetric T-junction device (Scale bar: $5 \mu \mathrm{m}$ ). $L_{1}$ and $L_{2}$ denote the length of the $\mathrm{TaS}_{2}$ arm above and below the junction, respectively. Right panel: Thickness profile of the $\mathrm{TaS}_{2}$ (in top) and $\mathrm{MoS}_{2}$ (in bottom) flakes along the white and yellow dashed arrows indicated in the optical image. (c)-(d) Current-voltage characteristics of $\mathrm{TaS}_{2} / \mathrm{MoS}_{2}$ junction $\left(\mathrm{T}_{1} \mathrm{M}\right)$ probed between terminals $\mathrm{T}_{1}$ and $\mathrm{M}$ (with terminal $\mathrm{T}_{2}$ open) as a function of $V_{g}$ varying from $-60 \mathrm{~V}$ to $50 \mathrm{~V}$ in steps of $10 \mathrm{~V}$ at $290 \mathrm{~K}$ [in (c)] and at $180 \mathrm{~K}$ [in (d)]. Inset of (c): For $V_{T_{1}}>0, \mathrm{Ni}$ acts as a source of electrons, while for $V_{T_{1}}<0, \mathrm{TaS}_{2}$ sources electrons into the $\mathrm{MoS}_{2}$ channel. Forward and reverse sweeps are indicated by black arrows. (e) Current-voltage characteristics of 2-probe $1 \mathrm{~T}-\mathrm{TaS}_{2}$ device (probed between terminals $\mathrm{T}_{1}$ and $\mathrm{T}_{2}$ ) depicting NC-IC phase transition at $298 \mathrm{~K}$. Inset: Crystal structure of $1 \mathrm{~T}-\mathrm{TaS}_{2}$ in $\mathrm{NC}$ phase (bottom left) and IC phase (top right). 

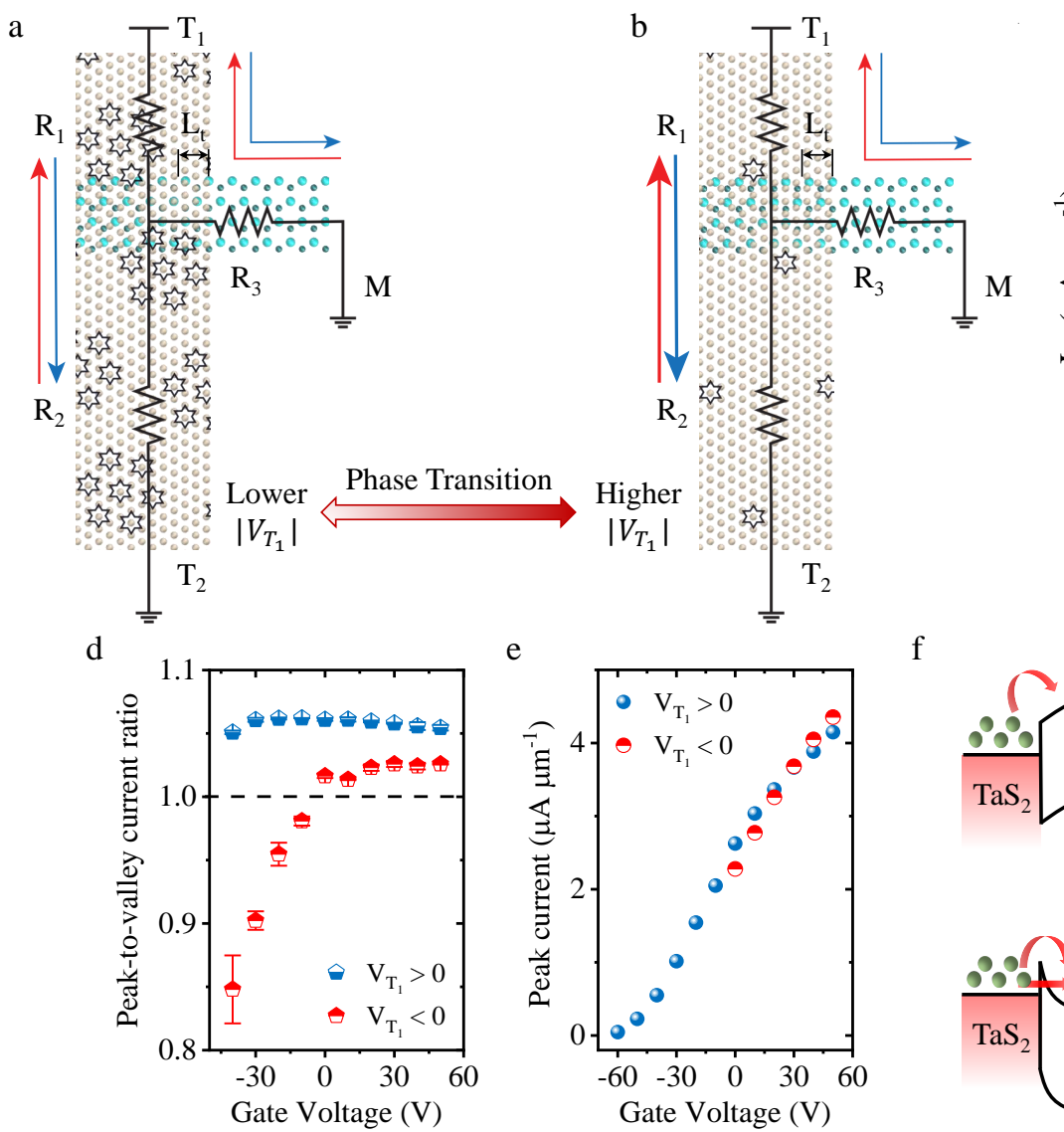

c
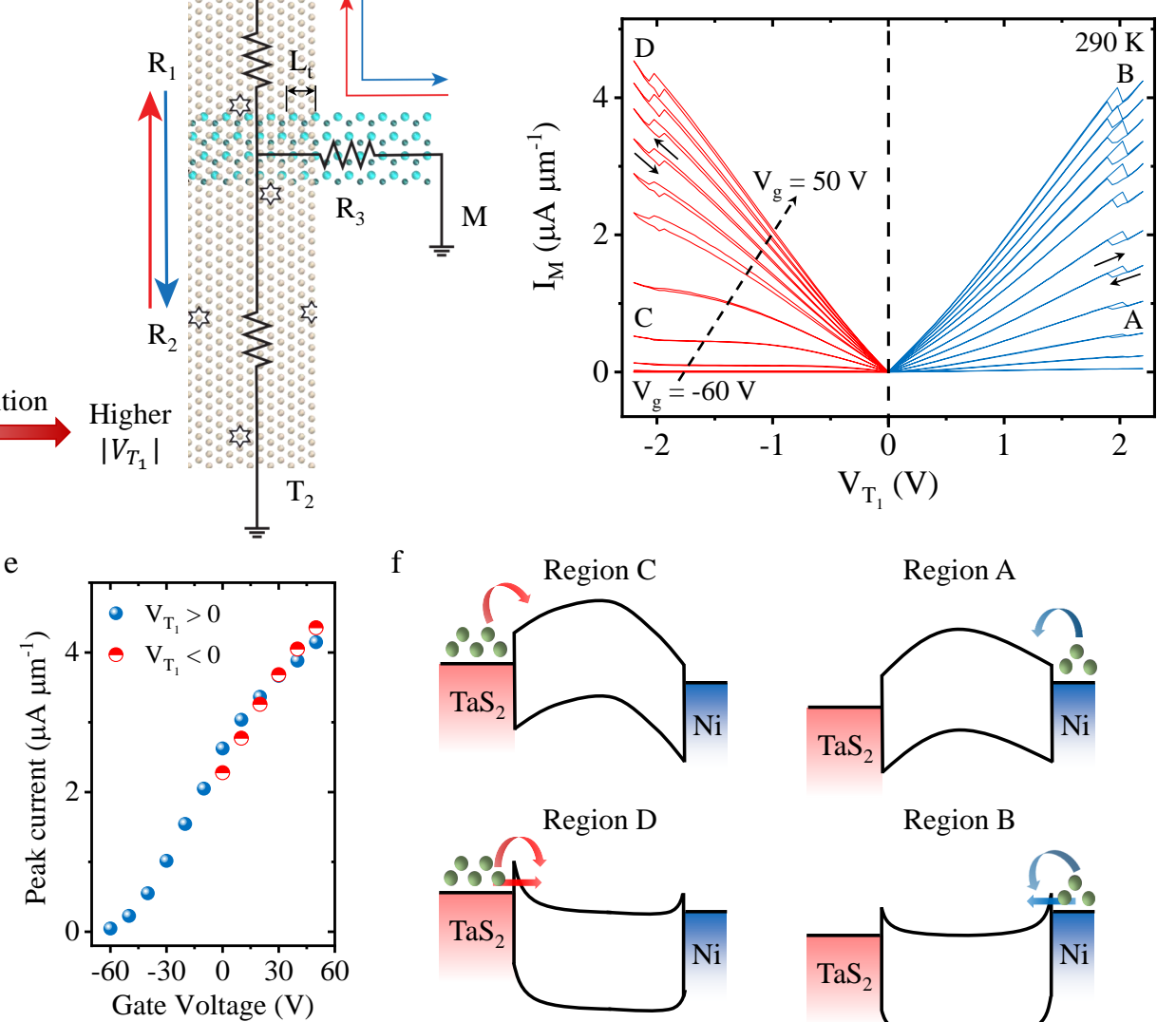

f
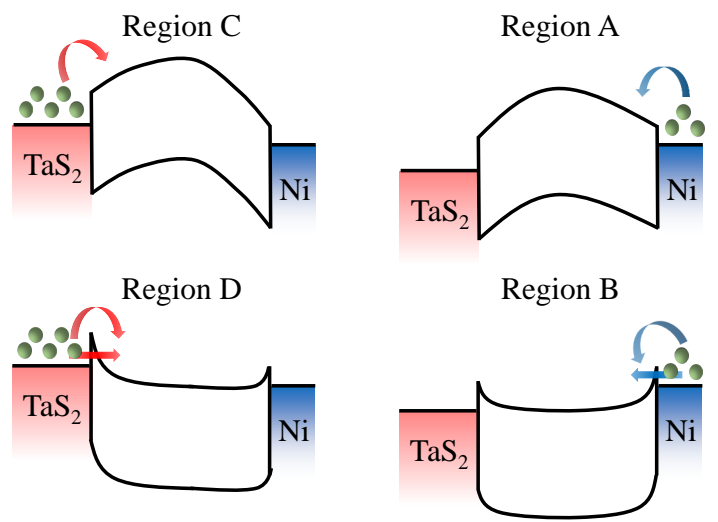

Figure 2: Gate tunable Negative Differential Resistance (NDR). (a)-(b) Equivalent circuit diagram of the device $\mathrm{D}_{1}$ embedded with crystal structure of $1 \mathrm{~T}-\mathrm{TaS}_{2}$ in NC-phase [in (a)] and IC-phase [in (b)]. Blue and red arrows indicate the direction of current flow when $V_{T_{1}}>0$ and $V_{T_{1}}<0$, respectively. (c) Current through the $\mathrm{MoS}_{2}$ branch $\left(I_{M}\right)$ versus $V_{T_{1}}$ as a function of $V_{g}$ varying from $-60 \mathrm{~V}$ to $50 \mathrm{~V}$ in steps of $10 \mathrm{~V}$ at $290 \mathrm{~K}$ depicting NDR for both $V_{T_{1}}>0$ and $V_{T_{1}}<0$. Forward and reverse sweeps are indicated by black arrows. (d) Peak-to-valley current ratio (PVCR) as the function of $V_{g}$ for both $V_{T_{1}}>0$ (bottom-filled blue pentagon) and $V_{T_{1}}<0$ (top-filled red pentagon) at $290 \mathrm{~K}$. (e) Peak current $\left(I_{p}\right)$ versus $V_{g}$ for both $V_{T_{1}}>0$ (blue spheres) and $V_{T_{1}}<0$ (half-filled red circles) at $290 \mathrm{~K}$. (f) Band diagram corresponding to different regions of operation, namely, A, B, C and D as indicated in (c). 
a
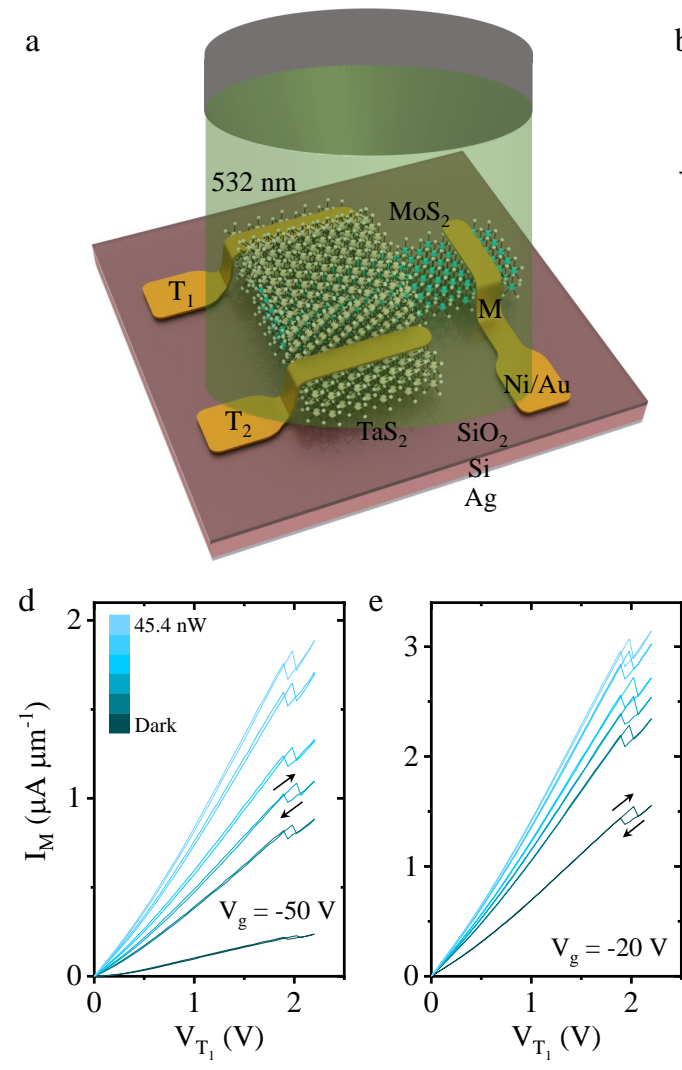

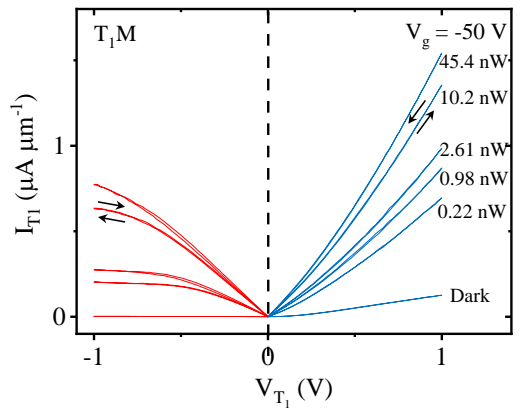

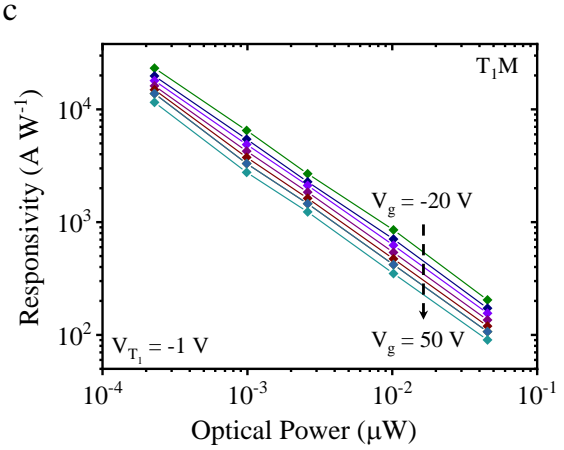
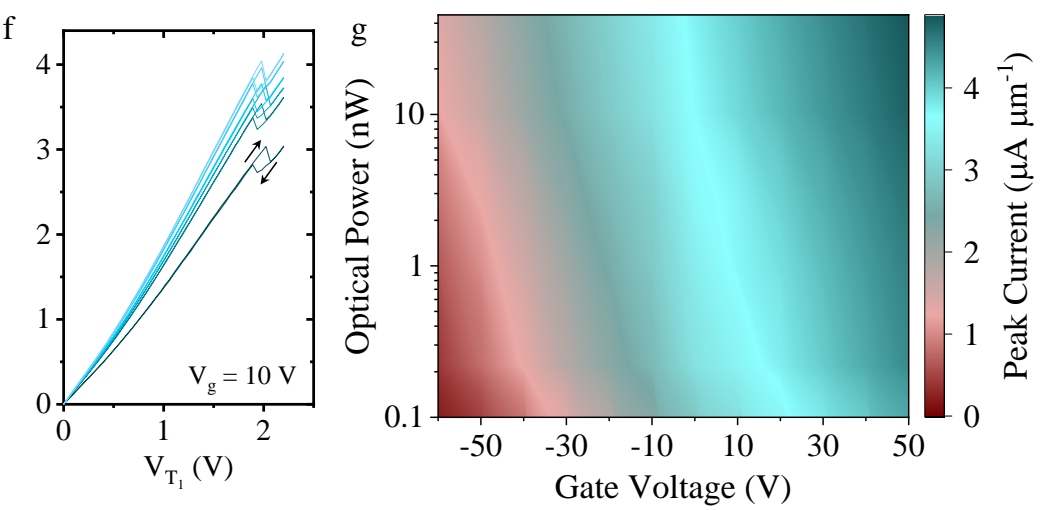

Figure 3: Light tunable NDR. (a) Schematic diagram of the device under photo-excitation. (b) Current-voltage characteristics of heterojunction $\mathrm{T}_{1} \mathrm{M}$ (with $\mathrm{T}_{2}$ open) at $V_{g}=-50 \mathrm{~V}$ as a function of $532 \mathrm{~nm}$ laser excitation power, showing strong photogating. (c) Responsivity versus excitation power at different $V_{g}$ values for $V_{T_{1}}=-1 V$. (d)-(f) $I_{M}$ versus $V_{T_{1}}$ as a function of excitation power at $V_{g}=-50 \mathrm{~V}$ [in (d)], $V_{g}=-20 \mathrm{~V}$ [in (e)] and $V_{g}=10 \mathrm{~V}$ [in (f)]. Forward and reverse sweeps are indicated by black arrows. (g) Color plot of peak current in gate voltage-excitation power control space. 

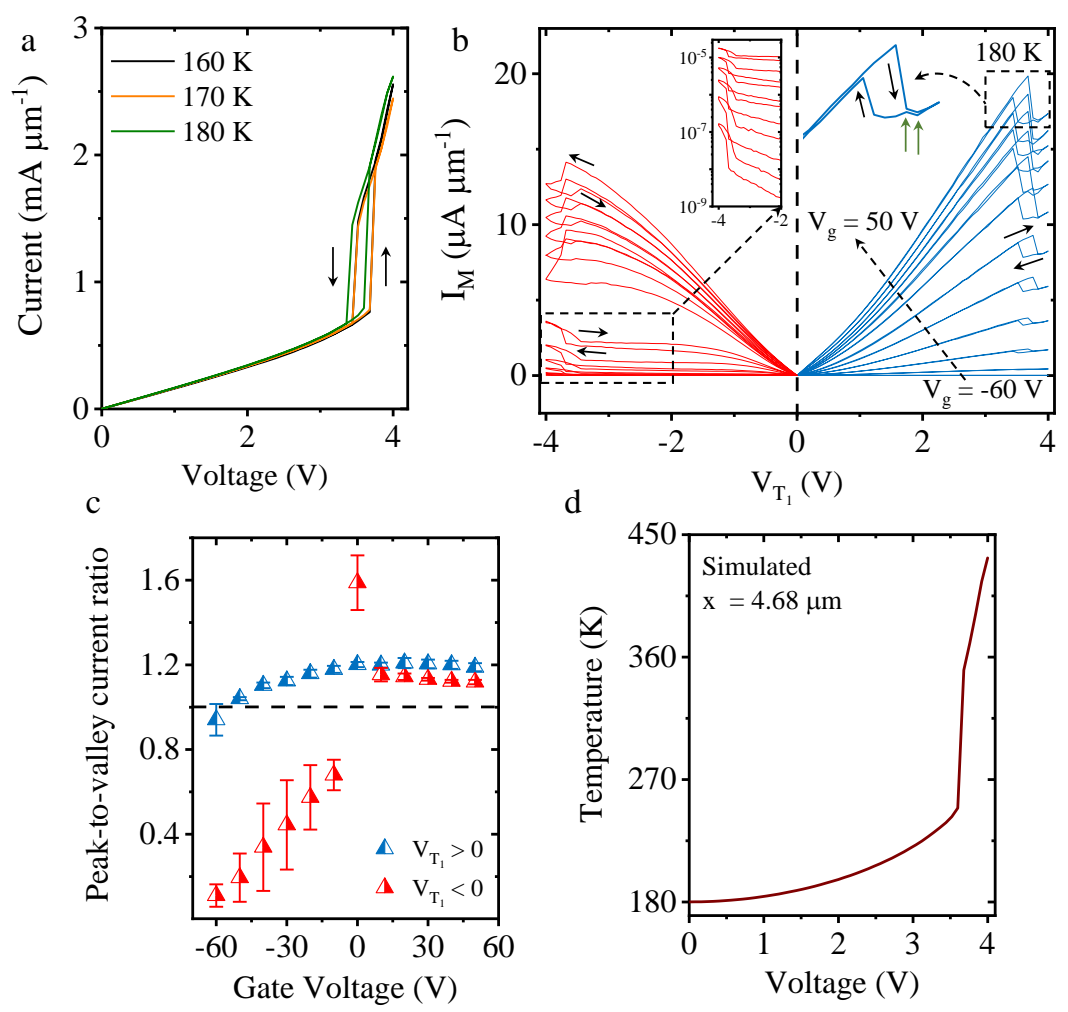

Figure 4: Enhancing PVCR at low temperature. (a) Current-voltage characteristics of 2-probe $\mathrm{TaS}_{2}$ (probed between terminals $\mathrm{T}_{1}$ and $\mathrm{T}_{2}$ ) at $160 \mathrm{~K}$ (black trace), $170 \mathrm{~K}$ (orange trace) and $180 \mathrm{~K}$ (olive trace). (b) $I_{M}$ versus $V_{T_{1}}$ as a function of $V_{g}$ varying from $-60 \mathrm{~V}$ to $50 \mathrm{~V}$ in steps of $10 \mathrm{~V}$ at $180 \mathrm{~K}$. Left Inset: $I_{M}$ versus $V_{T_{1}}$ for $V_{T_{1}}<0$ and $V_{g}<0$ in logarithmic scale, showing sharp enhancement in current during phase transition due to SBH suppression effect. Right Inset: Zoomed-in view of NDR region for $\mathrm{MoS}_{2}$ injection at $V_{g}=50 \mathrm{~V}$ clearly indicating multi-state induced NDR wherein different transitions are indicated by green arrows. Forward and reverse sweeps are indicated by black arrows. (c) PVCR versus $V_{g}$ for $V_{T_{1}}>0$ (left-filled blue triangles) and $V_{T_{1}}<0$ (right-filled red triangles) injection at $180 \mathrm{~K}$. (d) Simulated temperature variation in the middle of $\mathrm{TaS}_{2}$ channel $(x=4.68 \mu m)$ as a function of the voltage bias applied. 
Table 1: Performance benchmarking with existing literature

\begin{tabular}{|c|c|c|c|c|c|c|c|}
\hline Stack & Mechanism & $\begin{array}{c}\mathrm{T} \\
(\mathrm{K})\end{array}$ & $\begin{array}{c}I_{P} \\
(\mathbf{n A})\end{array}$ & $\begin{array}{c}J_{P} \\
\left(n A \mu m^{-2}\right)\end{array}$ & PVCR & $\begin{array}{c}\text { Gate } \\
\text { Control } \\
\text { of } \\
\text { NDR }\end{array}$ & $\begin{array}{c}\text { Light } \\
\text { Control } \\
\text { of } \\
\text { NDR }\end{array}$ \\
\hline \multirow{3}{*}{$\mathrm{MoS}_{2} / \mathrm{TaS}_{2}$ (This work) } & \multirow{3}{*}{ CDW } & 300 & $2.07 \times 10^{4}$ & $4.15 \times 10^{4}$ & 1.06 & \multirow{3}{*}{ Yes } & \multirow{3}{*}{ Yes } \\
\hline & & \multirow{2}{*}{180} & $9.92 \times 10^{4}$ & $1.98 \times 10^{5}$ & $\begin{array}{c}1.2 \\
@ V_{g}=50 \mathrm{~V}\end{array}$ & & \\
\hline & & & $4.8 \times 10^{4}$ & $9.61 \times 10^{4}$ & $\begin{array}{c}1.59 \\
@ V_{g}=0 V\end{array}$ & & \\
\hline \multirow{2}{*}{$\mathrm{BP} / \mathrm{SnSe}_{2} 2$} & \multirow{2}{*}{$\mathrm{BTBT}^{a}$} & 300 & 145 & 1.45 & 1.8 & \multirow{2}{*}{ Yes } & \multirow{2}{*}{ No } \\
\hline & & 80 & 175 & 1.75 & 2.8 & & \\
\hline $\mathrm{DG} \mathrm{MoS}_{2} / \mathrm{WSe}_{2} 21$ & BTBT & 77 & 1.26 & 0.45 & 1.68 & Yes & No \\
\hline $\mathrm{WSe}_{2} / \mathrm{SnSe}_{2}$ & BTBT & 77 & 0.27 & - & 1.03 & No & No \\
\hline $\mathrm{MoS}_{2} / \mathrm{WSe}_{2} 23$ & BTBT & 300 & 0.24 & - & 1.55 & Yes & No \\
\hline $\mathrm{Gr} / \mathrm{hBN} / \mathrm{WSe}_{2} / \mathrm{SnSe}_{2} 24$ & BTBT & 300 & $3 \times 10^{4}$ & $1.46 \times 10^{3}$ & 4 & Yes & No \\
\hline \multirow{2}{*}{$\mathrm{hBN} / \mathrm{Gr} / \mathrm{hBN} / \mathrm{Gr}^{25}$} & \multirow{2}{*}{$\mathrm{RTD}^{b}$} & 300 & 75 & 125 & 1.3 & \multirow{2}{*}{ Yes } & \multirow{2}{*}{ No } \\
\hline & & 7 & 110 & 183 & 3.6 & & \\
\hline $\mathrm{MoS}_{2} 26$ & RTD & 10 & 2.1 & - & 1.1 & Yes & No \\
\hline $\mathrm{Gr} / \mathrm{WSe}_{2} / \mathrm{MoS}_{2} 27$ & RTD & 300 & 0.05 & - & 1.9 & No & No \\
\hline $\mathrm{Gr} / \mathrm{MoSe}_{2} / \mathrm{WSe}_{2}$ & RTD & 300 & 0.08 & - & 2.2 & No & No \\
\hline \multirow{2}{*}{$\mathrm{BP} / \operatorname{ReS}_{2}$} & \multirow{2}{*}{ BTBT } & 300 & 2.7 & - & 4.2 & \multirow{2}{*}{ Yes } & \multirow{2}{*}{ No } \\
\hline & & 180 & 3 & - & 6.9 & & \\
\hline $\mathrm{Si} 32$ & BTBT & 300 & - & $4.25 \times 10^{1}$ & 5.05 & No & No \\
\hline $\mathrm{Si} 33$ & BTBT & 300 & - & $6.6 \times 10^{4}$ & 2.5 & No & No \\
\hline $\mathrm{Ge}^{34}$ & BTBT & 300 & - & 5.7 & 1.6 & No & No \\
\hline $\mathrm{Si} / \mathrm{SiGe}^{35}$ & $\operatorname{RITD}^{c}$ & 300 & $1.78 \times 10^{6}$ & $1 \times 10^{3}$ & 1.85 & No & No \\
\hline $\mathrm{Si} / \mathrm{SiGe} / \mathrm{Si}{ }^{36}$ & BTBT & 300 & - & $3 \times 10^{5}$ & 4.8 & No & No \\
\hline $\mathrm{GaN} / \mathrm{AlGaN}$ & RTD & 300 & - & $1 \times 10^{5}$ & 2 & No & No \\
\hline $\mathrm{AlN} / \mathrm{GaN}$ & RTD & 300 & $2.32 \times 10^{7}$ & $1.2 \times 10^{6}$ & 1.2 & No & No \\
\hline $\mathrm{Si} / \mathrm{SiGe}^{39}$ & RITD & 300 & - & $2.18 \times 10^{6}$ & 1.47 & No & No \\
\hline InAs/GaSb & BTBT & 300 & - & $2.2 \times 10^{7}$ & 3.9 & No & No \\
\hline
\end{tabular}

${ }^{a}$ Band-to-band tunneling; ${ }^{b}$ Resonant Tunneling; ${ }^{c}$ Resonant Interband Tunneling. 

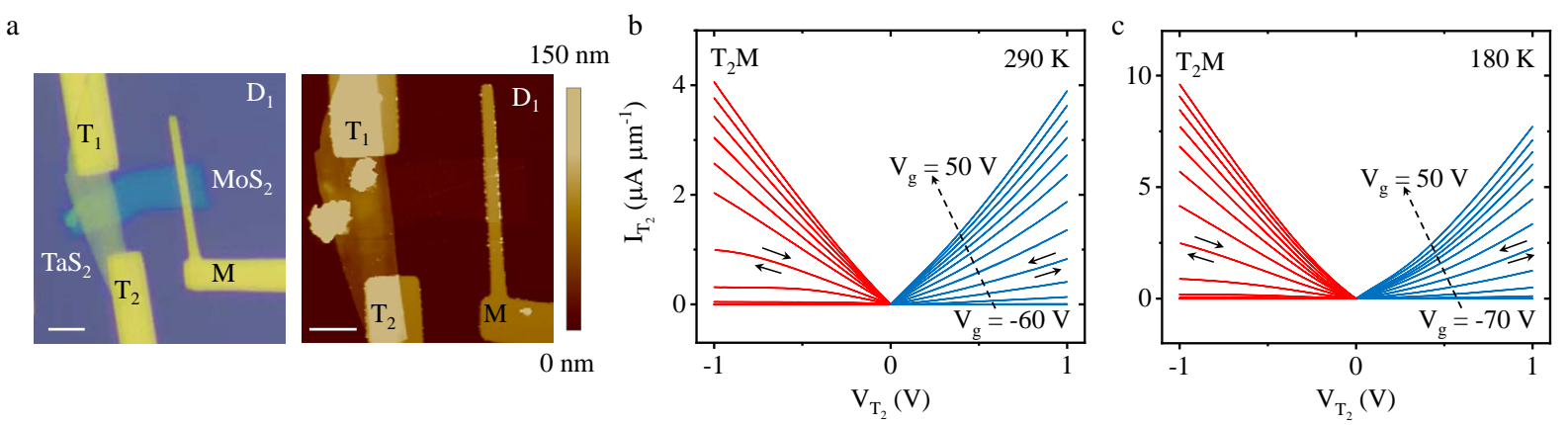

Figure S1: Electrical Characterization of $\mathrm{TaS}_{2} / \mathrm{MoS}_{2}$ junction. (a) Optical and AFM image of the device $\mathrm{D}_{1}$ in left and right panel respectively. Scale bar: $5 \mu \mathrm{m}$. (b)-(c) I-V characteristics of $\mathrm{TaS}_{2} / \mathrm{MoS}_{2}$ junction $\left(\mathrm{T}_{2} \mathrm{M}\right)$ of device $\mathrm{D}_{1}$ probed between terminals $\mathrm{T}_{2}$ and $\mathrm{M}$ (with terminal $\mathrm{T}_{1}$ open) as a function of back gate voltage $\left(V_{g}\right)$ at $290 \mathrm{~K}$ [in (a)] and at $180 \mathrm{~K}$ [in (b)] respectively. 
a

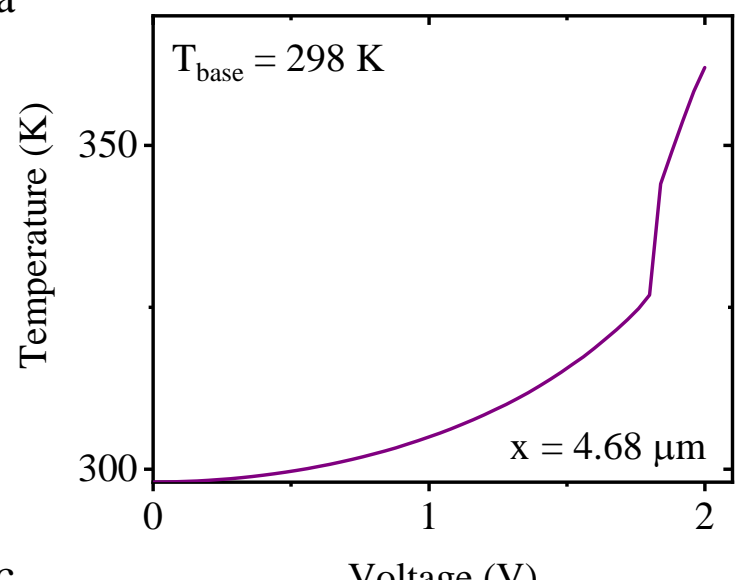

c

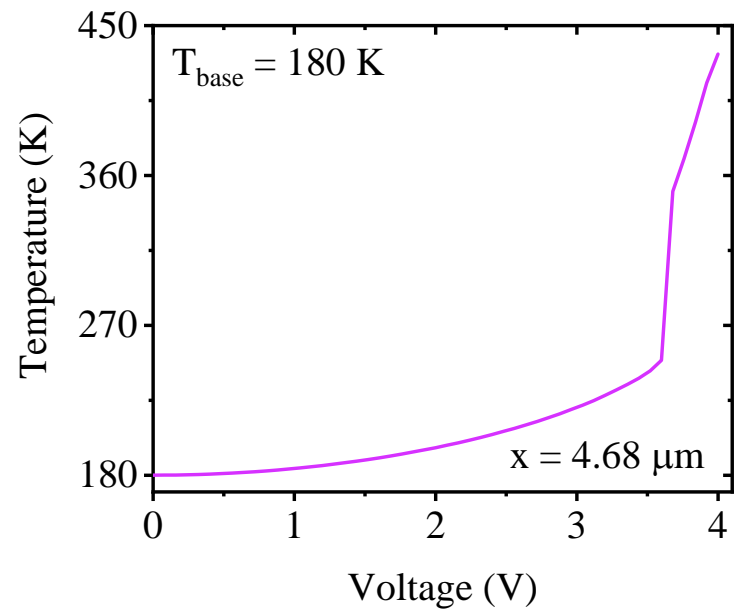

b
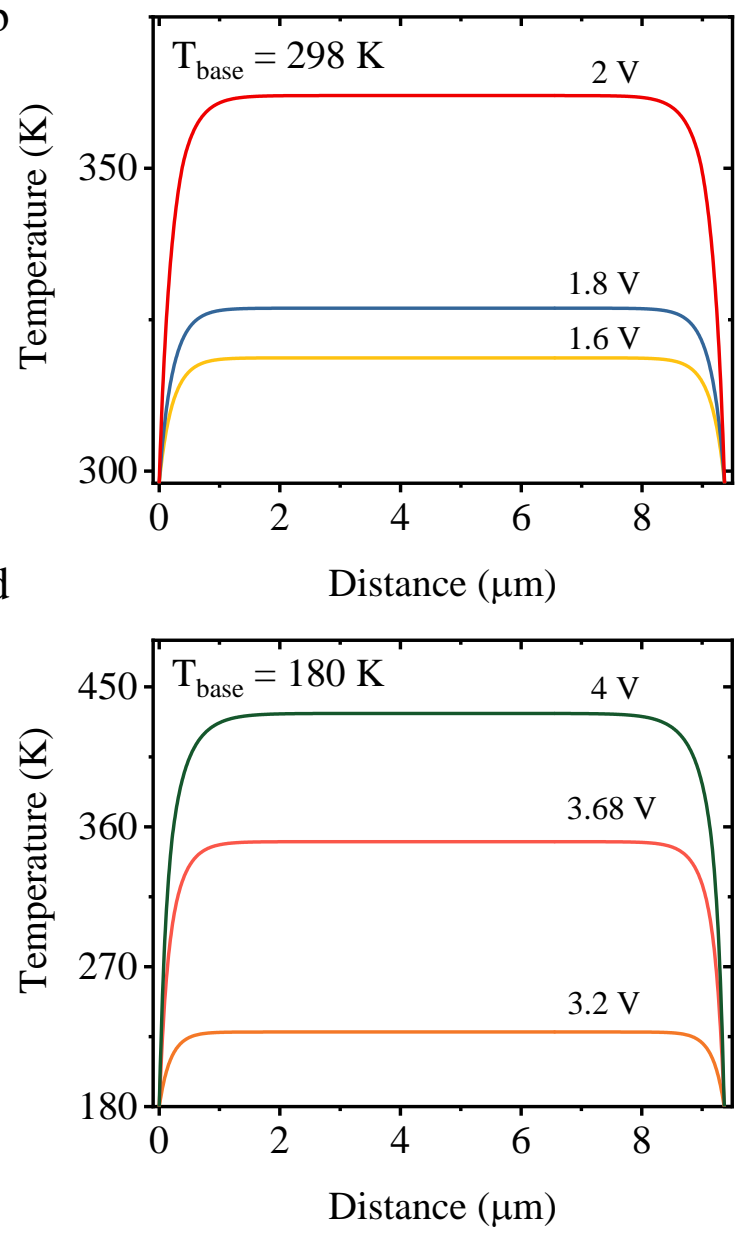

Figure S2: Simulated temperature variation along $\mathrm{TaS}_{\mathbf{2}}$ channel. (a)-(c) Simulated temperature profile at the middle of the $\mathrm{TaS}_{2}$ channel as the function of voltage for base temperature ( $T_{\text {base }}$ ) of $298 \mathrm{~K}$ [in (a)] and $180 \mathrm{~K}$ [in (c)]. (b) Simulated temperature variation along the $\mathrm{TaS}_{2}$ channel at bias voltage of $1.6 \mathrm{~V}$ (before transition), $1.8 \mathrm{~V}$ (at transition) and $2 \mathrm{~V}$ (after transition) for $T_{\text {base }}$ of $298 \mathrm{~K}$. (d) Simulated temperature variation along the $\mathrm{TaS}_{2}$ channel at bias voltage of $3.2 \mathrm{~V}$ (before transition), $3.68 \mathrm{~V}$ (at transition) and $4 \mathrm{~V}$ (after transition) for $T_{\text {base }}$ of $180 \mathrm{~K}$. 
a

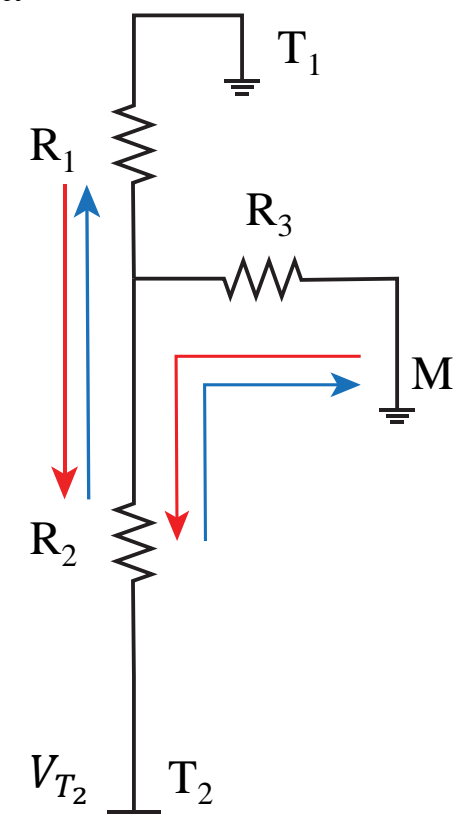

b

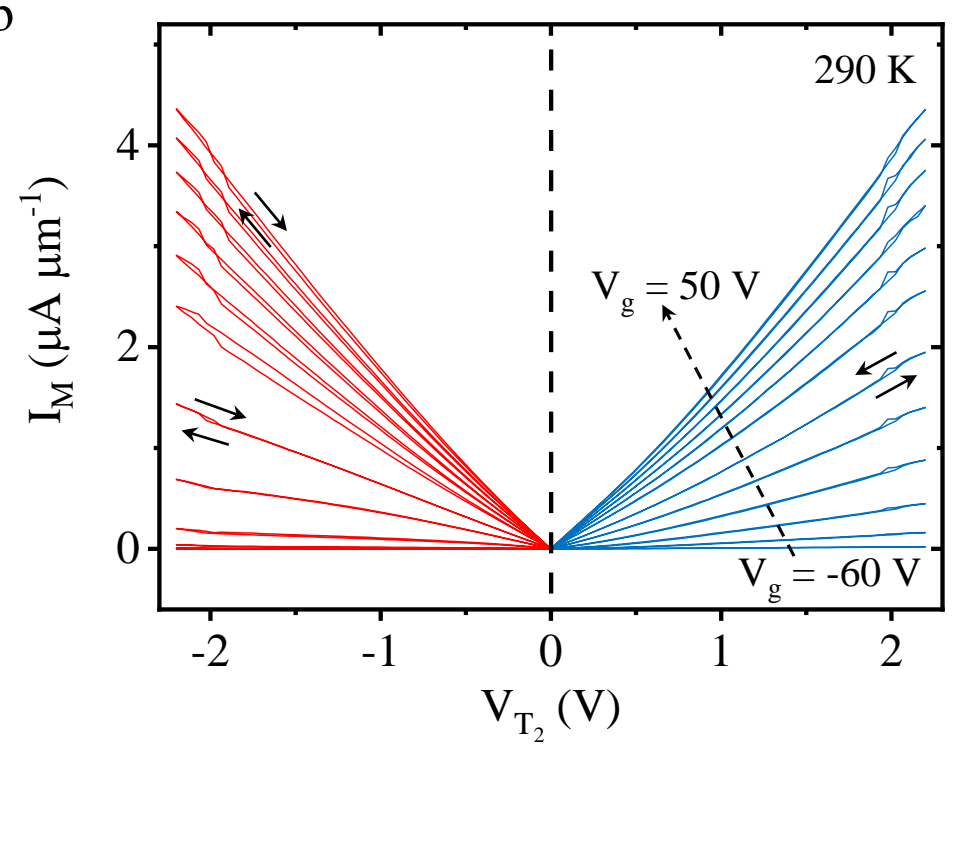

Figure S3: Characteristics for $\mathbf{T}_{\mathbf{2}}$ biasing in device $\mathbf{D}_{\mathbf{1}}$. (a) Equivalent circuit diagram of the 4-terminal device $\mathrm{D}_{1}$ when bias is applied at terminal $\mathrm{T}_{2}$ whereas terminal $\mathrm{T}_{1}$ and $\mathrm{M}$ are grounded. (b) $I_{M}$ versus $V_{T_{2}}$ as a function of back gate voltage $\left(V_{g}\right)$ varied from $-60 \mathrm{~V}$ to $50 \mathrm{~V}$ in steps of $10 \mathrm{~V}$ at $290 \mathrm{~K}$ depicting a small increment in current for both $V_{T_{1}}>0$ (blue traces) and $V_{T_{1}}<0$ (red traces). Forward and reverse sweeps are indicated by black arrows. No NDR is observed for this biasing condition, in agreement with Equation 1 in the main text. 
a
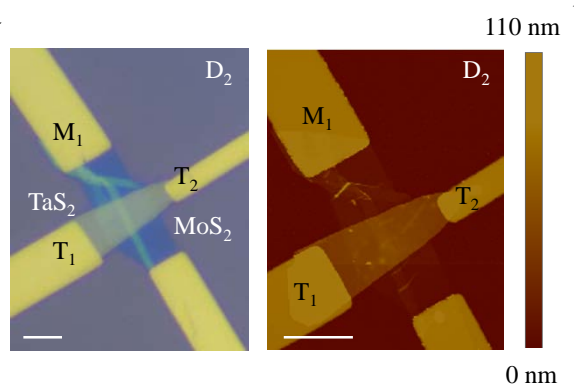

d

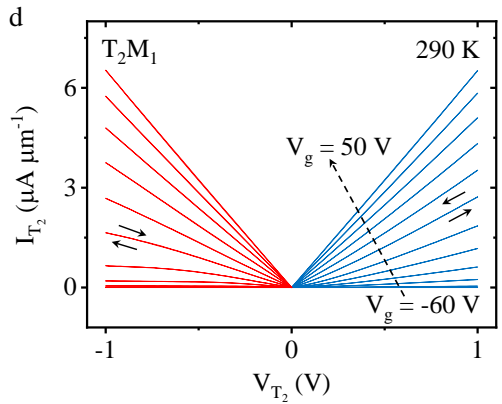

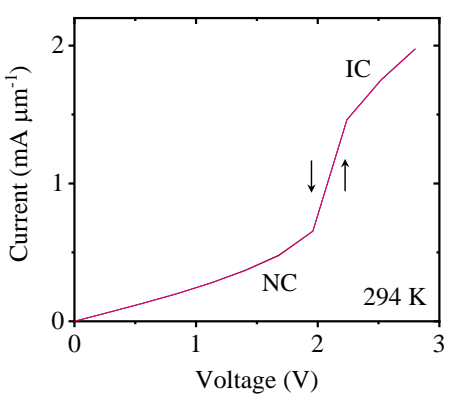

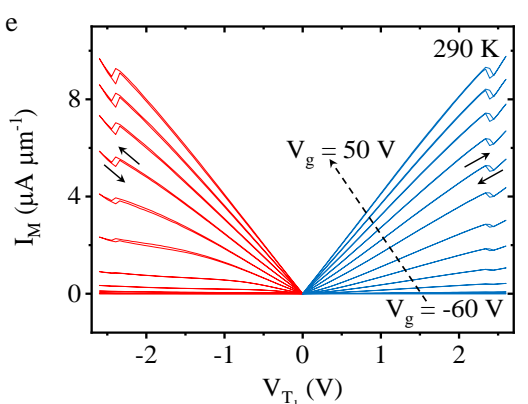

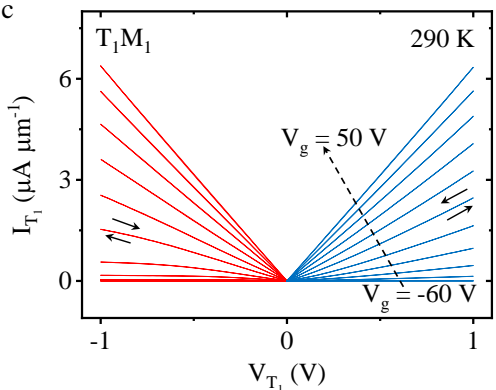

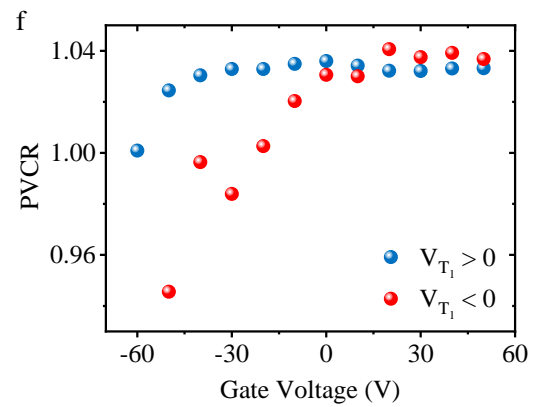

Figure S4: Gate tunable NDR with reduced hysteresis in $1 \mathrm{~T}-\mathrm{TaS}_{\mathbf{2}} / \mathbf{2 H}-\mathrm{MoS}_{2}$ asymmetric T-junction device $\left(\mathbf{D}_{2}\right)$. (a) Optical and AFM image of the fabricated device $\mathrm{D}_{2}$ in left and right panel respectively. Scale bar: $5 \mu \mathrm{m}$. (b) I-V characteristics of 2probe $1 \mathrm{~T}-\mathrm{TaS}_{2}$ device $\mathrm{D}_{2}$ (probed between terminals $\mathrm{T}_{1}$ and $\mathrm{T}_{2}$ ) under high field condition depicting NC-IC phase transition. (c) I-V characteristics of $\mathrm{TaS}_{2} / \mathrm{MoS}_{2}$ junction $\left(\mathrm{T}_{1} \mathrm{M}_{1}\right)$ probed between terminals $\mathrm{T}_{1}$ and $\mathrm{M}_{1}$ (with terminal $\mathrm{T}_{2}$ open) as a function of back gate voltage $\left(V_{g}\right)$ varied from $-60 \mathrm{~V}$ to $50 \mathrm{~V}$ in steps of $10 \mathrm{~V}$ at $290 \mathrm{~K}$. (d) I-V characteristics of $\mathrm{T}_{2} \mathrm{M}_{1}$ junction probed between terminals $\mathrm{T}_{2}$ and $\mathrm{M}_{1}$ (with terminal $\mathrm{T}_{1}$ open) for different $V_{g}$ at $290 \mathrm{~K}$. (e) $I_{M}$ versus $V_{T_{1}}$ as the function of back gate voltage $\left(V_{g}\right)$ at 290 $\mathrm{K}$ depicting NDR for both $V_{T_{1}}>0$ (blue traces) and $V_{T_{1}}<0$ (red traces). Forward and reverse sweeps are indicated by black arrows. Reduced hysteresis in the observed NDR is inherited by phase switching curve in (b). (f) Peak-to-valley current ratio (PVCR) as a function of gate voltage $\left(V_{g}\right)$ for both $V_{T_{1}}>0$ (blue spheres) and $V_{T_{1}}<0$ (red spheres). 
a

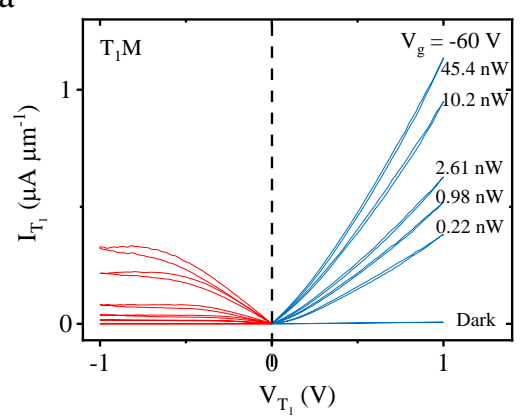

d

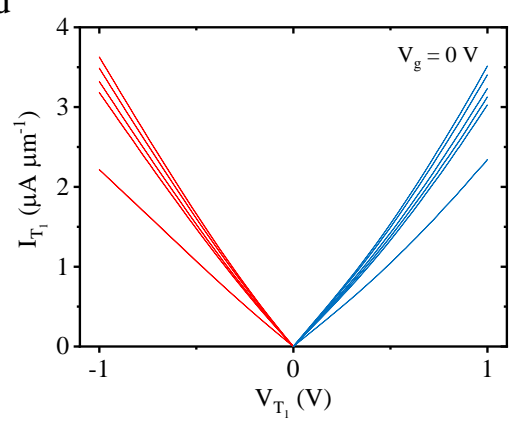

b

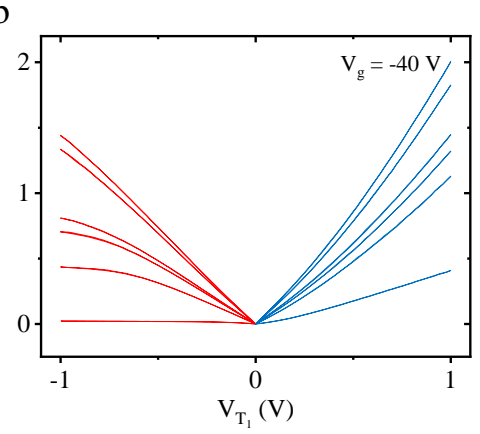

$\mathrm{e}$

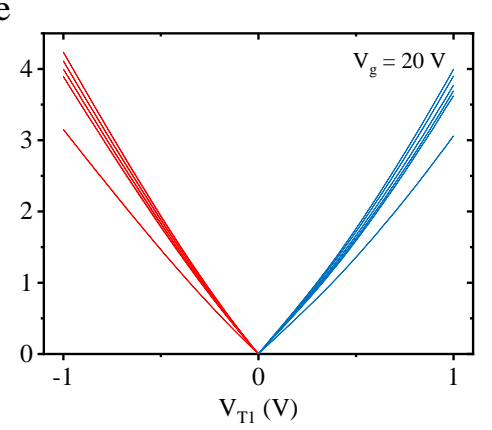

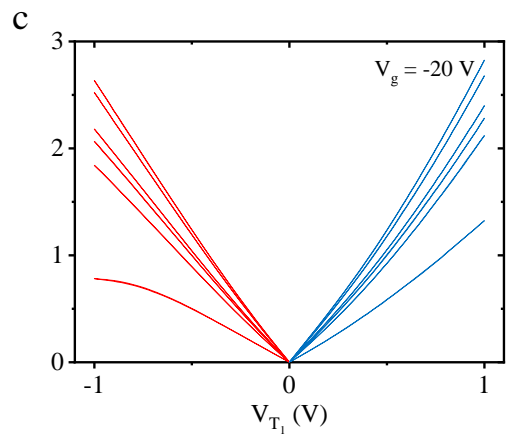

$\mathrm{f}$

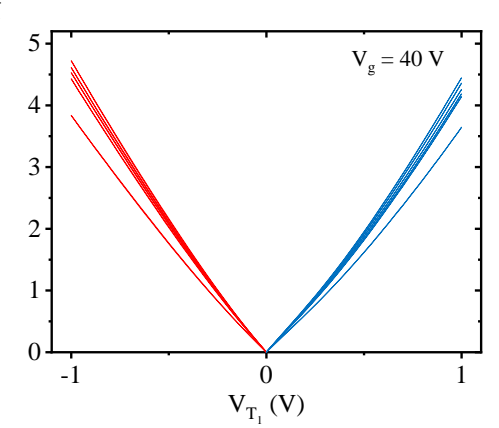

Figure S5: Light response of heterojunction $\mathbf{T}_{\mathbf{1}} \mathbf{M}$ of device $\mathbf{D}_{\mathbf{1}}$. (a)-(f) Currentvoltage characteristics of heterojuntion $\mathrm{T}_{1} \mathrm{M}$ of device $\mathrm{D}_{1}$ (probed between terminals $\mathrm{T}_{1}$ and $\mathrm{M}$ with terminal $\mathrm{T}_{2}$ open) with excitation wavelength of $532 \mathrm{~nm}$ at $V_{g}=-60 \mathrm{~V}$ [in (a)], $V_{g}=-40 \mathrm{~V}$ [in (b)], $V_{g}=-20 \mathrm{~V}$ [in (c)], $V_{g}=0 \mathrm{~V}$ [in (d)], $V_{g}=20 \mathrm{~V}$ [in (e)] and $V_{g}=40 \mathrm{~V}[$ in (f) $]$. 

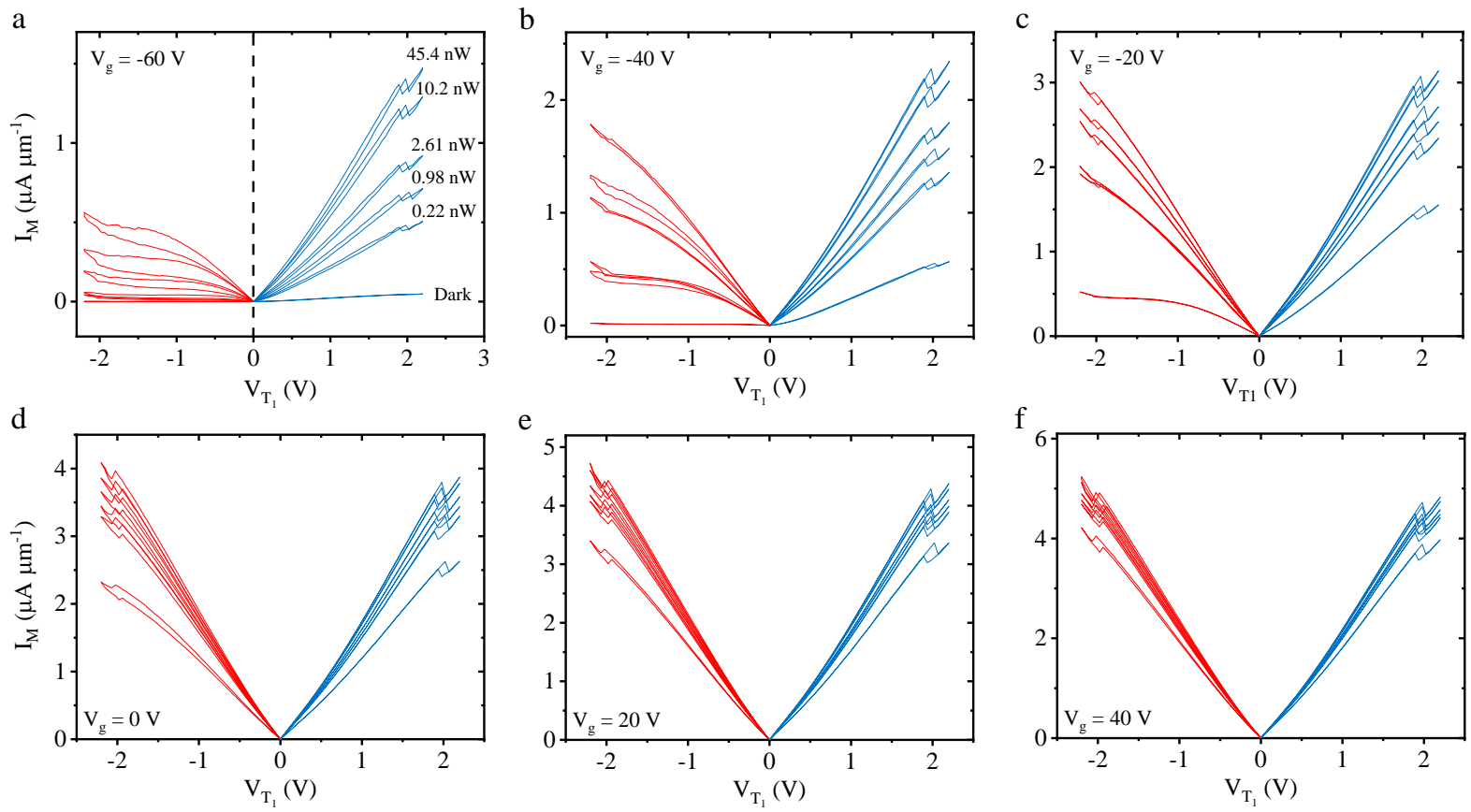

Figure S6: Light controlled NDR in $\mathrm{TaS}_{2} / \mathrm{MoS}_{2}$ asymmetric T-junction $\left(\mathrm{D}_{1}\right)$. (a)-(f) $I_{M}$ versus $V_{T_{1}}$ as the function of $532 \mathrm{~nm}$ laser excitation power at $V_{g}=-60 \mathrm{~V}$ [in (a)], $V_{g}=-40 \mathrm{~V}$ [in (b)], $V_{g}=-20 \mathrm{~V}$ [in (c)], $V_{g}=0 \mathrm{~V}$ [in (d)], $V_{g}=20 \mathrm{~V}$ [in (e)] and $V_{g}=40 \mathrm{~V}[$ in (f)]. 
a

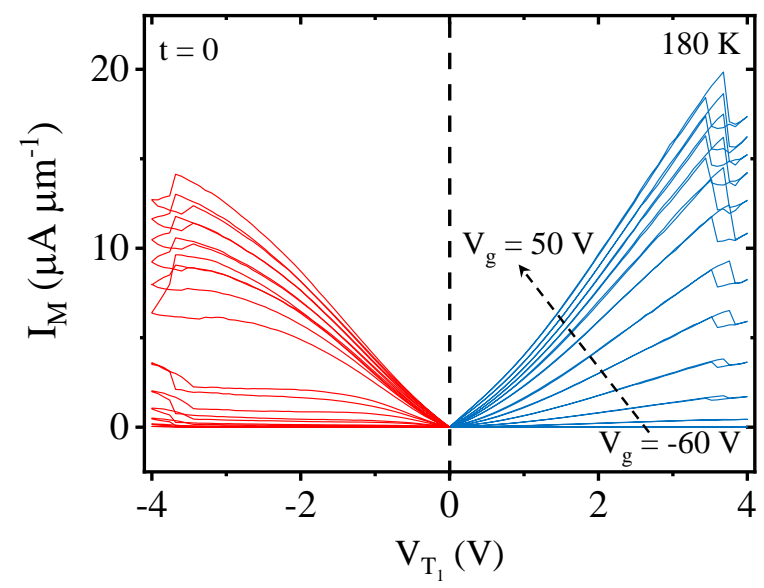

b

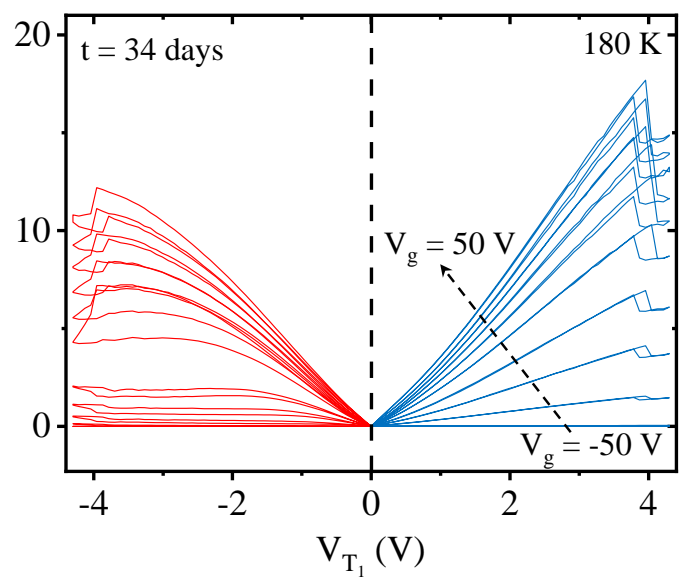

Figure S7: Stability of $\mathbf{T a S}_{\mathbf{2}} / \mathrm{MoS}_{\mathbf{2}}$ asymmetric T-junction $\left(\mathrm{D}_{1}\right)$. (a) $I_{M}$ versus $V_{T_{1}}$ as the function of back gate voltage $\left(V_{g}\right)$ varied from $-60 \mathrm{~V}$ to $50 \mathrm{~V}$ in steps of $10 \mathrm{~V}$ at $180 \mathrm{~K}$. (b) $I_{M}$ versus $V_{T_{1}}$ depicting NDR after 34 days under similar conditions as (a). 


\section{Graphical TOC Entry}

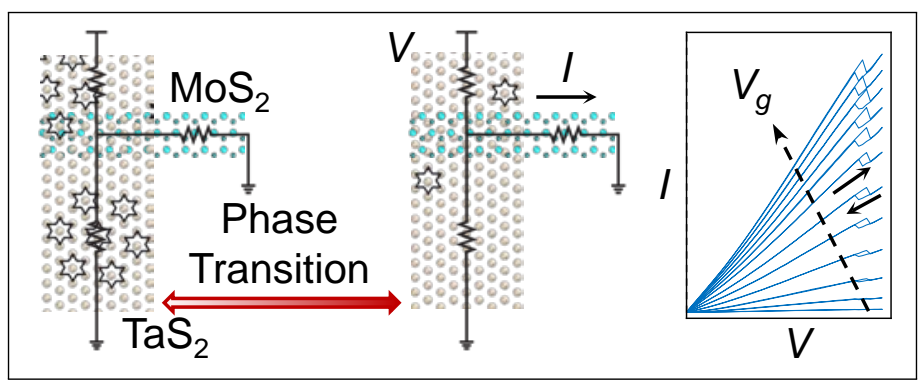

